\title{
WITT EQUIVALENCE OF FUNCTION FIELDS OVER GLOBAL FIELDS
}

\author{
PAWEŁ GŁADKI AND MURRAY MARSHALL
}

\begin{abstract}
Witt equivalent fields can be understood to be fields having the same symmetric bilinear form theory. Witt equivalence of finite fields, local fields and global fields is well understood. Witt equivalence of function fields of curves defined over archimedean local fields is also well understood. In the present paper, Witt equivalence of general function fields over global fields is studied. It is proved that for any two such fields $K, L$, any Witt equivalence $K \sim L$ induces a cannonical bijection $v \leftrightarrow w$ between Abhyankar valuations $v$ on $K$ having residue field not finite of characteristic 2 and Abhyankar valuations $w$ on $L$ having residue field not finite of characteristic 2 . The main tool used in the proof is a method for constructing valuations due to Arason, Elman and Jacob 11. The method of proof does not extend to non-Abhyankar valuations. The result is applied to study Witt equivalence of function fields over number fields. It is proved, for example, that if $k, \ell$ are number fields and $k\left(x_{1}, \ldots, x_{n}\right) \sim \ell\left(x_{1}, \ldots, x_{n}\right), n \geq 1$, then $k \sim \ell$ and the 2-ranks of the ideal class groups of $k$ and $\ell$ are equal.
\end{abstract}

\section{INTRODUCTION}

Let $K$ be a field. Denote by $W(K)$ the Witt ring of (non-degenerate) symmetric bilinear forms over $K$; see [27, 29] or [48] for the definition in case $\operatorname{char}(K) \neq 2$ and [19, 20] or [33] for the definition in the general case. Denote by $Q(K)$ the quadratic hyperfield of $K$; roughly speaking this is the same thing as the quadratic form scheme of $K$ 26] 27]; see Section 3 for the definition. We say two fields $K, L$ are Witt equivalent, denoted $K \sim L$, if $Q(K) \cong Q(L)$ as hyperfields, equivalently, if $W(K) \cong W(L)$ as rings; see Proposition 3.2 below. Witt equivalent fields can be understood as fields having the same symmetric bilinear form theory.

Witt equivalence of finite fields and local fields is well understood. Witt equivalence of global fields is considered in [5, 36, [42, 43, [44. Witt equivalence of function fields of curves defined over local and global fields is considered in [13, 21, 22. (Note, however, that there is a serious error in the proof of Theorem 1.3 in [21], in the proof of (1.3.1) $\Rightarrow(1.3 .2)$.)

It is well-known that any hyperfield isomorphism $\alpha: Q(K) \rightarrow Q(L)$ carries orderings of $K$ to orderings of $L$ in the sense that if $P \subseteq K^{*}$ is the positive cone of an ordering of $K$ then

$$
Q=\left\{s \in L^{*}: \bar{s}=\alpha(\bar{t}) \text { for some } t \in P\right\}
$$

2000 Mathematics Subject Classification. Primary 11E81, 12J20 Secondary 11E04, 11E12.

Key words and phrases. symmetric bilinear forms, quadratic forms, Witt equivalence of fields, function fields, global fields, valuations, Abhyankar valuations.

The research of the second author was supported in part by NSERC of Canada. 
is the positive cone of an ordering of $L$. Here, $\bar{x}$ denotes the image of $x$ under the canonical map $K^{*} \rightarrow K^{*} / K^{* 2}$. This correspondence can also be deduced from the fact that orderings on $K$ correspond to ring homomorphisms from $W(K)$ to $\mathbb{Z}$.

It is natural to wonder if a similar result holds for valuations, i.e., if the valuations of a field $K$ can be detected by looking at the quadratic hyperfield $Q(K)$. At this level of generality the result is false. E.g., $\mathbb{C} \sim \mathbb{F}_{2}$ and $\mathbb{C}((x)) \sim \mathbb{F}_{5}$. In each of these examples, the first field has lots of non-trivial valuations, but the second field has only the trivial valuation. At the same time, there is a detection procedure which works for certain sorts of fields. E.g., if $K, L$ are global fields of characteristic $\neq 2$, then any hyperfield isomorphism $\alpha: Q(K) \rightarrow Q(L)$ induces in a cannonical way a bijection $v \leftrightarrow w$ between valuations $v$ of $K$ and valuations $w$ of $L$; see [5, 36], [42, [43], 44. The main tool for setting up this bijection is a method of constructing valuations described in [1, which is based, in turn, on earlier constructions, of a similar sort, described in [15] and [46].

In the present paper we extend the above-mentioned result for global fields, proving that if $K, L$ are function fields over global fields then any hyperfield isomorphism $\alpha: Q(K) \rightarrow Q(L)$ induces in a canonical way a bijection $v \leftrightarrow w$ between Abhyankar valuations $v$ of $K$ having residue field not finite of characteristic 2 and Abhyankar valuations $w$ of $L$ having residue field not finite of characteristic 2; see Theorem 7.5.

Our results are applied to study Witt equivalence of function fields over number fields; see Corollary 8.2. Theorem 8.6 and Corollary 8.8. It is proved, for example, that if $k\left(x_{1}, \ldots, x_{n}\right) \sim \ell\left(x_{1}, \ldots, x_{n}\right)$, where $n \geq 1$ and $k$ and $\ell$ are number fields, then $k \sim \ell$ and the 2-ranks of the ideal class groups of $k$ and $\ell$ are equal.

In Sections 2 and 3 we recall basic terminology which is used throughout the paper. In Section 4 we establish basic connections between quadratic hyperfields and valuations. In Section 5 we apply the result in [1] to understand the behavior of valuations under Witt equivalence; see Theorem [5.3. In Section 6 we recall the terminology of function fields, global fields and Abhyankar valuations, and we introduce the idea of nominal transcendence degree.

The main new results in the paper are found in Sections 5,7 and 8.

The authors would like to express their thanks to the annonymous referee for his careful reading and useful improvements that made the presentation more comprehensible.

\section{HYPERFIELDS}

A hyperfield is an object like a field, but where the addition is allowed to be multivalued. Hyperfields were introduced by Krasner 223, 24], in connection with his work on valuations. Hyperfields were also introduced independently in 31 where they were called multifields.

A hyperfield is a system $(H,+, \cdot,-, 0,1)$ where $H$ is a set, + is a multivalued binary operation on $H$, i.e., a function from $H \times H$ to the set of all subsets of $H$, - is a binary operation on $H,-: H \rightarrow H$ is a function, and 0,1 are elements of $H$ such that

I. $(H,+,-, 0)$ is a canonical hypergroup, terminology as in Mittas 35, i.e.,

(1) $c \in a+b \Rightarrow a \in c+(-b)$,

(2) $a \in b+0$ iff $a=b$,

(3) $(a+b)+c=a+(b+c)$, and 
(4) $a+b=b+a$; and

II. $(H, \cdot, 1)$ is a commutative monoid, i.e., $(a b) c=a(b c), a b=b a$, and $a 1=a$ for all $a, b, c \in A$; and

III. $a 0=0$ for all $a \in H$; and

IV. $a(b+c) \subseteq a b+a c ;$ and

V. $1 \neq 0$ and every non-zero element has a multiplicative inverse.

Hyperfields form a category. A morphism from $H_{1}$ to $H_{2}$, where $H_{1}, H_{2}$ are hyperfields, is a function $\alpha: H_{1} \rightarrow H_{2}$ which satisfies $\alpha(a+b) \subseteq \alpha(a)+\alpha(b)$, $\alpha(a b)=\alpha(a) \alpha(b), \alpha(-a)=-\alpha(a), \alpha(0)=0, \alpha(1)=1$.

Here are some elementary consequences of the hyperfield axioms: (i) $-0=0$ (ii) $-(-a)=a$ (iii) $a+b \neq \emptyset$ (iv) $a(-b)=-(a b)(\mathrm{v})(-a)(-b)=a b$.

Every field is a hyperfield. The simplest non-trivial examples of hyperfields are the quotient hyperfields. If $T$ is a subgroup of $H^{*}$, where $H$ is a field or hyperfield, the quotient hyperfield $H /{ }_{m} T=(H / m T,+, \cdot,-, 0,1)$ is defined as follows: $H / m T$ is the set of equivalence classes with respect to the equivalence relation $\sim$ on $H$ defined by $a \sim b$ iff $a s=b t$ for some $s, t \in T$. The operations on $H /{ }_{m} T$ are the obvious ones induced by the corresponding operations on $H$ : Denote by $\bar{a}$ the equivalence class of $a$. Then $\bar{a} \in \bar{b}+\bar{c}$ iff $a s \in b t+c u$ for some $s, t, u \in T, \bar{a} \bar{b}=\overline{a b}$, $-\bar{a}=\overline{-a}$. Also, $0=\overline{0}$, and $1=\overline{1}$. The group of non-zero elements of $H / m T$ is $H^{*} / T$. The subscript $m$ here is used to indicate that $H /{ }_{m} T$ is a quotient modulo a multiplicative subgroup $T$ and was introduced in [31]: although we call $\mathrm{H} / \mathrm{m} T$ a quotient, its construction really resembles more that of a localisation, and the authors believe that denoting it simply by $H / T$ might be somewhat misleading.

The hyperfield associated to an ordered abelian group $\Gamma:=(\Gamma, \cdot, 1, \leq)$ is $\Gamma \cup$ $\{0\}:=(\Gamma \cup\{0\},+, \cdot,-, 0,1)$, where

$$
a+b:=\left\{\begin{array}{l}
b \text { if } a<b \\
a \text { if } b<a \\
{[0, a] \text { if } a=b}
\end{array},\right.
$$

$a \cdot 0=0 \cdot a:=0$ and $-a:=a$. Convention: $0<a$ for all $a \in \Gamma$.

A valuation on a field $K$ is just a morphism $v: K \rightarrow \Gamma \cup\{0\}$, for some ordered abelian group $\Gamma:=(\Gamma, \cdot, 1, \leq)$. If $\Gamma$ is the value group of $v$, i.e., if $v$ is surjective, then $v$ induces an isomorphism $\bar{v}: K /{ }_{m} U \rightarrow \Gamma \cup\{0\}$, where $U$ is the unit group of $v \sqrt{1}$

See 32 for an example of a hyperfield which is not realizable as a quotient hyperfield of a field.

We are mostly interested in one special example of a quotient hyperfield, namely the hyperfield $K /{ }_{m} K^{* 2}$, for a fixed field $K$, and its particular connection to symmetric bilinear forms over $K$. Observe that, for a field $K$, and for $z, a, b \in K$ the following equivalence holds true:

$$
z=a x^{2}+b y^{2} \text { for some } x, y \in K^{*} \text { if and only if } \bar{z} \in \bar{a}+\bar{b} \text { in } K / m K^{* 2} .
$$

\footnotetext{
${ }^{1}$ The foregoing example notwithstanding, in what follows we will always use the more standard additive notation for valuations, i.e., a valuation is a function $v: K \rightarrow \Gamma \cup\{\infty\}$, for some ordered abelian group $\Gamma:=(\Gamma,+, 0, \geq)$.
} 
It turns out that, in fact, a slightly more general equivalence holds true, at least when $K \neq \mathbb{F}_{2}, \mathbb{F}_{3}$, $\operatorname{char}(K) \neq 2$ (see Proposition 3.1 below for details), namely:

$$
z=a x^{2}+b y^{2} \text { for some } x, y \in K \text { if and only if } \bar{z} \in \bar{a}+\bar{b} \text { in } K /{ }_{m} K^{* 2} \text {. }
$$

This equivalence fails to hold without these additional assumptions. Here it is necessary to modify the definition of addition in $K /{ }_{m} K^{* 2}$, defining $\bar{a}+\bar{b}$, for $a, b \neq$ 0 , "by hand". Fortunately enough, this can be also done more conceptually, by defining a new addition on any given hyperfield.

If $H=(H,+, \cdot,-, 0,1)$ is a hyperfield, the prime addition on $H$ is defined by

$$
a+{ }^{\prime} b= \begin{cases}a+b & \text { if one of } a, b \text { is zero } \\ a+b \cup\{a, b\} & \text { if } a \neq 0, b \neq 0, b \neq-a . \\ H & \text { if } a \neq 0, b \neq 0, b=-a\end{cases}
$$

In the next section we use the following result:

Proposition 2.1. For any hyperfield $H:=(H,+, \cdot,-, 0,1), H^{\prime}:=\left(H,+^{\prime}, \cdot,-, 0,1\right)$ is also a hyperfield.

Proof. We make use of the fact that $a+b \subseteq a+^{\prime} b$. I (1) Suppose $c \in a+^{\prime} b$. If $c \in a+b$ then $a \in c+(-b) \subseteq c+^{\prime}(-b)$. Otherwise, $a, b \neq 0$ and $c=a$ or $c=b$ or $a=-b$. In each of these cases, $a \in c+^{\prime}(-b)$ is clear. (2) Since $b+^{\prime} 0=b+0$ this is clear. (3) As explained in [31, Lemma 1.3], it suffices to show $\left(a+^{\prime} b\right)+^{\prime} c \subseteq a+^{\prime}\left(b+{ }^{\prime} c\right)$, i.e., if $x \in y+{ }^{\prime} c$ for some $y \in a+{ }^{\prime} b$ then $x \in a+{ }^{\prime} z$ for some $z \in b+^{\prime} c$. If $x \in y+c$ and $y \in a+b$ this is clear. Otherwise either $y, c \neq 0$ and $(x=y$ or $x=c$ or $y=-c)$ or $a, b \neq 0$ and $(y=a$ or $y=b$ or $a=-b$ ). In the first case, if $x=y$ take $z=b$ if $b \neq 0$ and $z=c$ if $b=0$; if $x=c$ take $z=c$; if $y=-c$ take $z=-a$ if $a \neq 0$ and $z=x$ if $a=0$. In the second case, if $y=a$ take $z=c$ if $c \neq 0$ and $z=b$ if $c=0$; if $y=b$ take $z=x$ if $x \neq 0$ and $z=-a$ if $x=0$; if $a=-b$ take $z=b$. (4) is clear. II, III and V are clear. IV Suppose $x \in b+{ }^{\prime} c$. If $x \in b+c$ then $a x \in a(b+c) \subseteq a b+a c \subseteq a b+^{\prime} a c$. Otherwise, $b, c \neq 0$ and $x=b$ or $x=c$ or $b=-c$. In each of these cases $a x \in a b+^{\prime} a c$ is clear.

We refer to $H^{\prime}$ as the prime of the hyperfield $H$. Observe that if $T$ is a subgroup of $H^{*}$ then $H^{\prime} /{ }_{m} T=(H / m T)^{\prime}$.

\section{Quadratic hyperfields AND Witt equivalence}

Let $K$ be a field. The quadratic hyperfield of $K$, denoted $Q(K)$, is defined to be the prime of the hyperfield $K /{ }_{m} K^{* 2} 2$ Note that $Q(K)^{*}=K^{*} / K^{* 2}$.

Proposition 3.1. Assume $\bar{a} \in Q(K)^{*}$. Then

(1) $\bar{a}^{2}=\overline{1}$.

(2) If $\bar{a} \neq-\overline{1}$ then $\overline{1}+\bar{a}$ is a subgroup of $Q(K)^{*}{ }^{3}$

\footnotetext{
${ }^{2}$ This is the same object referred to in 31. page 458]. Roughly speaking, it is the quadratic form scheme of $K$, terminology as in [26] or [27, with zero adjoined.

${ }^{3}$ If $G=(G,-1, V)$ is an (abstract) quadratic form scheme, terminology as in 26], then $H=(H,+, \cdot,-, 0,1)$, where $H:=G \cup\{0\}$,

$$
a+b:=\left\{\begin{array}{l}
a \text { if } b=0 \\
b \text { if } a=0 \\
a \cdot V(a b) \text { if } a, b \neq 0, b \neq-a \\
H \text { if } a, b \neq 0, b=-a
\end{array}\right.
$$
}


(3) If $K \neq \mathbb{F}_{3}, \mathbb{F}_{5}$ and $\operatorname{char}(K) \neq 2$ then $Q(K)=K /{ }_{m} K^{* 2}$.

Proof. (1) $a \in K^{*}$ so $a^{2} \in K^{* 2}$. It follows that $\bar{a}^{2}=\overline{a^{2}}=\overline{1}$. (2) If $\overline{0} \in \overline{1}+\bar{a}$, then $\bar{a} \in \overline{0}+(-\overline{1})$, so $\bar{a}=-\overline{1}$, which contradicts our assumption. This proves $\overline{1}+\bar{a} \subseteq K^{*} / K^{* 2}$. Clearly $\overline{1} \in \overline{1}+\bar{a}$. Each $\bar{b} \in K^{*} / K^{* 2}$ satisfies $\bar{b} \bar{b}=\overline{b^{2}}=\overline{1}$, so is its own inverse. Closure of $\overline{1}+\bar{a}$ under multiplication follows from the standard identity

$$
\left(x_{1}^{2}+a y_{1}^{2}\right)\left(x_{2}^{2}+a y_{2}^{2}\right)=\left(x_{1} x_{2}-a y_{1} y_{2}\right)^{2}+a\left(x_{1} y_{2}+x_{2} y_{1}\right)^{2} .
$$

(3) It suffices to show $\forall b \in K, b \in a K^{* 2}-a K^{* 2}$. Scaling, we are reduced to the case $a=1$. If $b \neq \pm 1$, the identity $b=\left(\frac{b+1}{2}\right)^{2}-\left(\frac{b-1}{2}\right)^{2}$ shows that $b \in K^{* 2}-K^{* 2}$. Thus we are reduced to showing $\pm 1 \in K^{* 2}-K^{* 2}$. Scaling, we are reduced further to showing $1 \in K^{* 2}-K^{* 2}$. Since $K \neq \mathbb{F}_{3}, \mathbb{F}_{5}$, and char $K \neq 2,\left|K^{*}\right| \geq 6$, so there exists $b \in K^{*}, b^{2} \neq \pm 1$. Then $b^{2}=\left(\frac{b^{2}+1}{2}\right)^{2}-\left(\frac{b^{2}-1}{2}\right)^{2}$, so, dividing by $b^{2}$, $1 \in K^{* 2}-K^{* 2}$.

The interest in $Q(K)$ stems from its connection to symmetric bilinear forms over $K$. One is mainly interested in the characteristic $\neq 2$ case. In this case, symmetric bilinear forms and quadratic forms are the same thing.

Denote by $W(K)$ the Witt ring of non-degenerate symmetric bilinear forms over $K$; see [27, 29] or [48] for the definition in case char $(K) \neq 2$ and [19, 20] or 33 . for the definition in the general case.

A (non-degenerate diagonal) binary form over $K$ is just an ordered pair $\langle\bar{a}, \bar{b}\rangle$, $\bar{a}, \bar{b} \in K^{*} / K^{* 2}$. The value set of such a form, denoted by $D_{K}\langle\bar{a}, \bar{b}\rangle$, is the set of non-zero elements of $\bar{a}+\bar{b}$, i.e., $D_{K}\langle\bar{a}, \bar{b}\rangle$ is the image under $K^{*} \rightarrow K^{*} / K^{* 2}$ of the subset $D_{K}\langle a, b\rangle$ of $K^{*}$ defined by

$$
D_{K}\langle a, b\rangle:=\left\{\begin{array}{l}
K^{*} \text { if }-a b \in K^{* 2} \\
\left\{z \in K^{*}: z=a x^{2}+b y^{2}, x, y \in K\right\} \text { otherwise }
\end{array} .\right.
$$

Two binary forms $\langle\bar{a}, \bar{b}\rangle$ and $\langle\bar{c}, \bar{d}\rangle$ are considered to be equivalent, denoted $\langle\bar{a}, \bar{b}\rangle \approx$ $\langle\bar{c}, \bar{d}\rangle$, if $\bar{c} \in D_{K}\langle\bar{a}, \bar{b}\rangle$ and $\bar{a} \bar{b}=\bar{c} \bar{c}$.

In terms of generators and relations, $W(K)$ is the integral group ring $\mathbb{Z}\left[K^{*} / K^{* 2}\right]$ factored by the ideal generated by $[1]+[-1]$ and all elements

$$
[a]+[b]-[c]-[d] \text { such that } \bar{a}, \bar{b}, \bar{c}, \bar{d} \in K^{*} / K^{* 2},\langle\bar{a}, \bar{b}\rangle \approx\langle\bar{c}, \bar{d}\rangle .
$$

See [20, Theorem 1.16 (iv) and Corollary 1.17] for the proof. Here, $[x]$ denotes the image of $\bar{x}$ under the canonical embedding $K^{*} / K^{* 2} \hookrightarrow \mathbb{Z}\left[K^{*} / K^{* 2}\right]$.

A hyperfield isomorphism $\alpha: Q(K) \rightarrow Q(L)$, where $K, L$ are fields, can be viewed as a group isomorphism $\alpha: K^{*} / K^{* 2} \rightarrow L^{*} / L^{* 2}$ such that $\alpha(-\overline{1})=-\overline{1}$ and

$$
\alpha\left(D_{K}\langle\bar{a}, \bar{b}\rangle\right)=D_{L}\langle\alpha(\bar{a}), \alpha(\bar{b})\rangle \text { for all } \bar{a}, \bar{b} \in K^{*} / K^{* 2},
$$

or, equivalently, as a group isomorphism $\alpha: K^{*} / K^{* 2} \rightarrow L^{*} / L^{* 2}$ which induces a ring isomorphism between $W(K)$ and $W(L)$. We say two fields $K$ and $L$ are Witt

$a \cdot 0=0 \cdot a:=0$ and $-a:=(-1) \cdot a$, is hyperfield satisfying (1) and (2) of Proposition 3.1 i.e., for all $a \in H^{*}$ (1) $a^{2}=1$ and (2) if $a \neq-1$ then $1+a$ is a subgroup of $H^{*}$. Conversely, every hyperfield $H$ satisfying (1) and (2) arises in this way, from some unique quadratic form scheme $G$. See [26] Theorem 1.4] for some equivalent descriptions of quadratic form schemes. The question of whether every quadratic form scheme is realized as the quadratic form scheme of a field appears to be still open. 
equivalent, denoted $K \sim L$, to indicate that $Q(K)$ and $Q(L)$ are isomorphic as hyperfields. For completeness and clarity we record the following:

Proposition 3.2. $K \sim L$ iff $W(K)$ and $W(L)$ are isomorphic as rings.

Proof. See 14 for the characteristic $\neq 2$ case. As remarked in [4], the Hauptsatz in 2] holds for all characteristics. The general case follows from this fact; see 29, Proposition 4.6].

For fields of characteristic $\neq 2$, Witt equivalence is also characterized in terms of Galois groups; see [34, Theorem 3.8].

It is well-known that the Witt ring of a field $K$ encodes the theory of symmetric bilinear forms over $K$. Witt equivalent fields can be understood as fields having the same symmetric bilinear form theory. The quadratic hyperfield $Q(K)$ encodes exactly the same information as the Witt ring $W(K)$. At the same time, it is a much simpler and easier object to deal with.

Hyperfields provide a first-order axiomatization of the algebraic theory of quadratic forms. Although other first-order descriptions have been already known for some time (see [9] and [30]), it seems that the theory of hyperfields is the most natural and the most easily understood. All the results presented in what follows can be "translated" to the traditional notion of Witt rings, and, as of today, the authors are not familiar with any results in the algebraic theory of quadratic forms that can be proven with the use of hyperfields, but can not be proven without them. Still, the authors believe that hyperfields make the exposition easier to read and to understand.

\section{QUADRATIC HYPERFIELDS AND VALUATIONS}

Let $H_{1}, H_{2}$ be hyperfields. Each morphism $\iota: H_{1} \rightarrow H_{2}$ induces a morphism $\bar{\iota}: H_{1} /{ }_{m} \Delta \rightarrow H_{2}$ where $\Delta:=\left\{x \in H_{1}^{*}: \iota(x)=1\right\}$. The morphism $\iota$ is said to be a quotient morphism if $\iota$ is an isomorphism, equivalently, if $\iota$ is surjective, and $\iota(c) \in \iota(a)+\iota(b)$ iff $c s \in a t+b u$ for some $s, t, u \in \Delta$. A morphism $\iota: H_{1} \rightarrow H_{2}$ is said to be a group extension if $\iota$ is injective, every $x \in H_{2}^{*} \backslash \iota\left(H_{1}^{*}\right)$ is rigid in the sense that $1+x \subseteq\{1, x\}$, and $y \in H_{1}, y \neq-1 \Rightarrow \iota(1+y)=1+\iota(y)$.

We assume now that $K$ is a field. For a valuation $v$ on $K, \Gamma_{v}$ denotes the value group, $A_{v}$ denotes the valuation ring, $M_{v}$ the maximal ideal, $U_{v}$ the unit group, and $K_{v}$ the residue field. $\pi=\pi_{v}: A_{v} \rightarrow K_{v}$ denotes the canonical homomorphism, i.e., $\pi(a)=a+M_{v}$. We say $v$ is discrete rank one if $\Gamma_{v}=\mathbb{Z}$. See [10, [12, [37, for background material on valuations.

We will be interested in the subgroup $T=\left(1+M_{v}\right) K^{* 2}$ of $K^{*}$.

Proposition 4.1. Suppose $v$ is non-trivial and $T=\left(1+M_{v}\right) K^{* 2}$. Then:

(1) $T \cup x T \subseteq T+x T$ for all $x \in K^{*}$;

(2) $T-T=K$;

(3) The map $Q(K) \rightarrow K / m T$ defined by $\bar{x} \mapsto x T$ is a quotient morphism.

Proof. (1) Pick $p \in K^{*}$ so that $v\left(p^{2}\right)>\max \{v(x),-v(x)\}$. Since we are assuming $v$ is non-trivial this is always possible. Then $t=1+p^{2} x \in T$, so $1=\frac{1}{t}\left(1+p^{2} x\right) \in$ $T+x T$, and, similarly, $p^{2}+x=x\left(1+\frac{p^{2}}{x}\right) \in x T$, so $x \in T+x T$. (2) Suppose $y \in K$.

\footnotetext{
${ }^{4}$ We are interested here in the case where the groups $H_{1}^{*}, H_{2}^{*}$ have exponent 2. In this situation, $1+x \subseteq\{1, x\} \Leftrightarrow 1+x=\{1, x\}$.
} 
Pick $p \in K^{*}$ so that $v\left(p^{2}\right)<v(y)$. Then $y=p^{2}\left(1+\frac{y}{p^{2}}\right)-p^{2} \in T-T$. (3) In view of (1) and (2), $K /{ }_{m} T=(K / m T)^{\prime}$, so this is clear.

Propositions 4.2 and 4.3 below are variants of old results of Springer [40, 41] couched in the language of quadratic hyperfields. Consider the canonical group isomorphism $\alpha: U_{v} K^{* 2} /\left(1+M_{v}\right) K^{* 2} \rightarrow K_{v}^{*} / K_{v}^{* 2}$ induced by $x \in U_{v} \mapsto \pi(x) \in K_{v}^{*}$. Define $\iota: Q\left(K_{v}\right) \rightarrow K / m T$ by $\iota(0)=0$ and $\iota(a)=\alpha^{-1}(a)$ for $a \in K_{v}^{*} / K_{v}^{* 2}$.

Proposition 4.2. Suppose $v$ is non-trivial and $T=\left(1+M_{v}\right) K^{* 2}$. Then:

(1) $\iota$ is a morphism;

(2) $\iota$ is a group extension.

Note: The cokernel of the group embedding $\alpha^{-1}: K_{v}^{*} / K_{v}^{* 2} \rightarrow K^{*} / T$ is equal to $K^{*} / U_{v} K^{* 2} \cong \Gamma_{v} / 2 \Gamma_{v}$. For this reason we sometimes say that $K /{ }_{m} T$ is a group extension of $Q\left(K_{v}\right)$ by the group $\Gamma_{v} / 2 \Gamma_{v}$.

Proof. (1) $\iota(a b)=\iota(a) \iota(b), \iota(-a)=-\iota(a), \iota(0)=0$ and $\iota(1)=1$ are clear. It remains to show $\iota(a+b) \subseteq \iota(a)+\iota(b)$. This is clear if one of $a, b$ is zero, so we can assume $a, b \neq 0$. Scaling, we are reduced to showing $\iota(1+a) \subseteq 1+\iota(a)$ for all $a \in K_{v}^{*} / K_{v}^{* 2}$. Represent $a$ by an element $\pi(x), x \in U_{v}$. Suppose $\pi(y)=$ $\pi(p)^{2}+\pi(q)^{2} \pi(x), p, q \in U_{v}, y \in A_{v}$. Then $y=p^{2}+q^{2} x+z, v(z)>0$, so $y=p^{2}\left(1+\frac{z}{p^{2}}\right)+q^{2} x \in T+T x$. In view of parts (1) and (2) of Proposition 4.1, this proves (1).

(2) Clearly $\iota$ is injective. Suppose $y=t_{1}+t_{2} x, t_{1}, t_{2} \in T, x \notin U_{v} K^{* 2}$. Then $v\left(t_{1}\right) \neq v\left(t_{2} x\right)$. If $v\left(t_{1}\right)<v\left(t_{2} x\right)$, then $y=t_{1}\left(1+\frac{t_{2} x}{t_{1}}\right) \in T$. If $v\left(t_{1}\right)>v\left(t_{2} x\right)$, then $y=t_{2} x\left(1+\frac{t_{1}}{t_{2} x}\right) \in T x$. This proves the rigidity assertion. Suppose now that $y=t_{1}+t_{2} x, t_{1}, t_{2} \in T, x \in U_{v}, \pi(x) \notin-K_{v}^{* 2}$. We want to show $\exists y^{\prime} \in$ $T y \cap U_{v}$ such that $\pi\left(y^{\prime}\right) \in K_{v}^{* 2}+K_{v}^{* 2} \pi(x)$ or $\pi\left(y^{\prime}\right) \in K_{v}^{* 2}$ or $\pi\left(y^{\prime}\right) \in K_{v}^{* 2} \pi(x)$. If $v(y)>\min \left\{v\left(t_{1}\right), v\left(t_{2} x\right)\right\}$ then $x \in-T$, which contradicts $\pi(x) \notin-K_{v}^{* 2}$. Thus $v(y)=\min \left\{v\left(t_{1}\right), v\left(t_{2} x\right)\right\}$. If $v\left(t_{1}\right) \leq v\left(t_{2}\right)$ take $y^{\prime}=\frac{y}{t_{1}}$. If $v\left(t_{1}\right)>v\left(t_{2}\right)$ take $y^{\prime}=\frac{y}{t_{2}}$.

Proposition 4.3. Suppose $v$ is non-trivial, $\operatorname{char}\left(K_{v}\right) \neq 2$, and $T=\left(1+M_{v}\right) K^{* 2}$. Then $K / m T$ is naturally identified with $Q\left(\tilde{K}_{v}\right)$, where $\tilde{K}_{v}$ denotes the henselization of $(K, v)$.

Note: The conclusions of Propositions 4.1, 4.2 and 4.3 also hold when $v$ is trivial, provided $K \neq \mathbb{F}_{3}, \mathbb{F}_{5}$ and $\operatorname{char}(K) \neq 2$.

Proof. Denote by $\tilde{v}$ the extension of $v$ to $\tilde{K}_{v}$. Since $\left(\tilde{K}_{v}, \tilde{v}\right)$ is henselian and $\operatorname{char}\left(K_{v}\right) \neq 2,1+M_{\tilde{v}} \subseteq \tilde{K}_{v}^{* 2}$. It follows that the embedding $K \hookrightarrow \tilde{K}_{v}$ induces a group homomorphism $\tau: K^{*} / T \rightarrow \tilde{K}_{v}^{*} / \tilde{K}_{v}^{* 2}$. Since $\left(\tilde{K}_{v}, \tilde{v}\right)$ is an immediate extension of $(K, v)$, one sees that $\tau$ is an group isomorphism. The image of $Q\left(K_{v}\right)^{*}$ in $K^{*} / T$ under the group extension $\iota: Q\left(K_{v}\right) \hookrightarrow K /{ }_{m} T$ is identified via $\tau$ with the image of $Q\left(K_{v}\right)^{*}$ in $Q\left(\tilde{K}_{v}\right)^{*}$ under the group extension $\iota: Q\left(K_{v}\right) \hookrightarrow Q\left(\tilde{K}_{v}\right)$. The conclusion follows from this.

If $v$ is discrete rank one, one can replace henselization by completion in Proposition 4.3. The assumption in Proposition 4.3 that $\operatorname{char}\left(K_{v}\right) \neq 2$ is crucial. One says that $v$ is dyadic if $\operatorname{char}(K)=0, \operatorname{char}\left(K_{v}\right)=2$. The structure of $Q\left(\tilde{K}_{v}\right)$ when $v$ is dyadic is complicated; see [27] or [29] for the case where $K$ is a number field and [16] and [17 for the case where $K$ is arbitrary. 
Remark 4.4. Suppose $v, v^{\prime}$ are valuations on $K$ with $v \preceq v^{\prime}$, i.e., $v^{\prime}$ is a coarsening of $v$, i.e., $A_{v} \subseteq A_{v^{\prime}}$. Then $M_{v^{\prime}} \subseteq M_{v}$ so $\left(1+M_{v^{\prime}}\right) K^{* 2} \subseteq\left(1+M_{v}\right) K^{* 2}$. Denote by $\bar{v}$ the valuation on $K_{v^{\prime}}$ induced by $v$, i.e., $\bar{v}\left(\pi_{v^{\prime}}(a)\right)=v(a)$, for $a \in U_{v^{\prime}}$. Note that $\bar{v}$ and $v$ have the same residue field. See [37, Chapter $\mathrm{C}]$ for background. Assume now that $v, v^{\prime}$ are non-trivial and that $v^{\prime}$ is a proper coarsening of $v$. Then $K / m\left(1+M_{v}\right) K^{* 2}$ is a group extension of the hyperfield $K_{v^{\prime}} / m\left(1+M_{\bar{v}}\right) K_{v^{\prime}}^{* 2}$ in a natural way, and the following diagram of hyperfields and hyperfield morphisms is commutative:

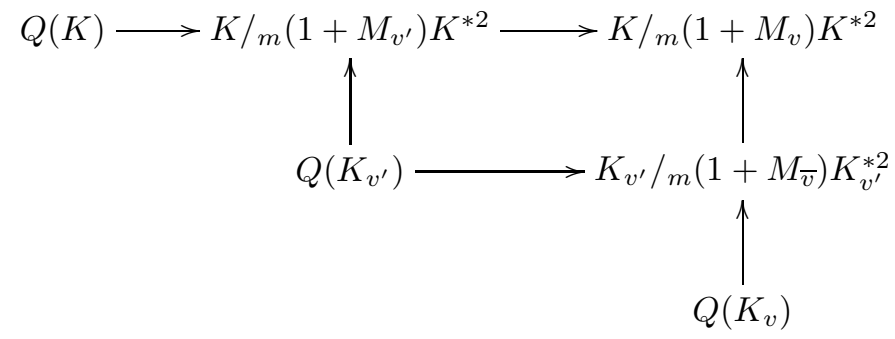

Here, the horizontal arrows are quotient morphisms and the vertical arrows are group extensions.

Let $T$ be a subgroup of $K^{*}$. We say $x \in K^{*}$ is $T$-rigid if $T+T x \subseteq T \cup T x$.

$$
B(T):=\left\{x \in K^{*}: \text { either } x \text { or }-x \text { is not } T \text {-rigid }\right\} \text {. }
$$

Elements of $B(T)$ are said to be $T$-basic. Note that if $x \in K^{*}$ is $T$-rigid and $y=t x$, $t \in T$, then $y$ is $T$-rigid. Consequently, $B(T)$ is a union of cosets of $T$. -1 is not $T$-rigid (because $0 \in T-T$ ), so $\pm T \subseteq B(T)$. We say that $T$ is exceptional if $B(T)= \pm T$ and either $-1 \in T$ or $T$ is additively closed.

We recall the result of Arason, Elman and Jacob alluded to in the introduction:

Theorem 4.5. Let $T \subseteq K^{*}$ be a subgroup and $H \subseteq K^{*}$ be a subgroup containing $B(T)$. Then there exists a subgroup $\hat{H}$ of $K^{*}$ such that $H \subseteq \hat{H}$ and $(\hat{H}: H) \leq 2$ and a valuation $v$ of $K$ such that $1+M_{v} \subseteq T$ and $U_{v} \subseteq \hat{H}$. Moreover, $\hat{H}=H$ works, unless $T$ is exceptional.

Proof. See [1, Theorem 2.16].

We will apply Theorem 4.5 to study Witt equivalence of function fields over global fields. We make frequent use of the following:

\section{Proposition 4.6.}

(1) $B\left(K^{* 2}\right)$ is a subgroup of $K^{*}$.

(2) Suppose $T=\left(1+M_{v}\right) K^{* 2}$ for some non-trivial valuation $v$ of $K$. Then $B(T) \subseteq U_{v} K^{* 2}$ and

$$
B(T)=\left\{x \in K^{*}: \bar{x}=\iota(\bar{y}) \text { for some } y \in B\left(K_{v}^{* 2}\right)\right\},
$$

where $\iota: Q\left(K_{v}\right) \hookrightarrow K / m T$ is the morphism in Proposition 4.2 $B(T)$ is a group and the group isomorphism $\iota: K_{v}^{*} / K_{v}^{* 2} \rightarrow U_{v} K^{* 2} / T$ induces a group isomorphism $B\left(K_{v}^{* 2}\right) / K_{v}^{* 2} \rightarrow B(T) / T$. $T$ is exceptional iff $K_{v}^{* 2}$ is exceptional.

Proof. (1) This is due to L. Berman. See [29, Theorem 5.18] for the proof. (2) The fact that $B(T)$ is a group follows from the fact that $B\left(K_{v}^{* 2}\right)$ is a group. The remaining assertions in (2) are a straightforward consequence of Proposition 4.2 . 


\section{Matching VAluations}

For any abelian group $\Gamma$, the rational rank of $\Gamma$, $\operatorname{denoted} \operatorname{rk}_{\mathbb{Q}}(\Gamma)$, is defined to be the dimension of the $\mathbb{Q}$-vector space $\Gamma \otimes_{\mathbb{Z}} \mathbb{Q}$.

We apply Theorem 4.5 to obtain useful results concerning the behaviour of valuations under Witt equivalence; refer to Theorem 5.3 below. We begin with two lemmas.

Lemma 5.1. If $\Gamma$ is a torsion free abelian group and $|\Gamma / 2 \Gamma|=2^{r}$, then $\operatorname{rk}_{\mathbb{Q}}(\Gamma) \geq r$.

This is well known. Observe that if $\Gamma \cong \mathbb{Z} \times \cdots \times \mathbb{Z}$ ( $r$ factors $)$ then $|\Gamma / 2 \Gamma|=2^{r}$, so $\operatorname{rk}_{\mathbb{Q}}(\Gamma)=r$ holds in this case. On the other hand, if $\Gamma=\mathbb{Q}$ for example then $\operatorname{rk}_{\mathbb{Q}}(\Gamma)=1, r=0$.

Proof. We claim that if $\alpha_{1}, \ldots, \alpha_{r} \in \Gamma$ are such that the $\operatorname{cosets} \alpha_{i}+2 \Gamma, i=1, \ldots, r$ are $\mathbb{F}_{2}$-linearly independent, then the $\alpha_{i}, i=1, \ldots, r$ are $\mathbb{Q}$-linearly independent. Suppose not. Then $\exists k_{i} \in \mathbb{Z}$ not all zero such that $\sum k_{i} \alpha_{i}=0$. Dividing by a suitable power of 2 , we can assume at least one of the $k_{i}$ is odd. This contradicts the assumption.

Lemma 5.2. Suppose $v, w$ are non-comparable valuations on a field $K$ and $\Gamma_{v}$ is finitely generated as an abelian group. Then $\left(1+M_{w}\right) K^{* 2} \nsubseteq\left(1+M_{v}\right) K^{* 2}$.

Note: Since the abelian group $\Gamma_{v}$ is torsion free, the assumption that $\Gamma_{v}$ is finitely generated is equivalent to $\Gamma_{v} \cong \mathbb{Z} \times \cdots \times \mathbb{Z}, r$ times, for some $r \geq 0$.

Proof. Denote by $u$ the finest common coarsening of $v$ and $w$ and by $\bar{v}$ and $\bar{w}$ the valuations on $K_{u}$ induced by $v$ and $w$ respectively. Since $\Gamma_{\bar{v}}$ is a subgroup of $\Gamma_{v}$, $\Gamma_{\bar{v}}$ is also finitely generated. Replacing $K$ by $K_{u}$ and $v$ and $w$ by $\bar{v}$ and $\bar{w}$, we are reduced to the case where $v$ and $w$ are independent. Fix $p \in K^{*}$ with $v(p) \notin 2 \Gamma_{v}$. By the approximation theorem there exists $x \in K$ such that $v(x-p)>v(p)$ and $w(x-1)>0$. Then $x \in 1+M_{w}$, and $v(x)=v(p) \notin 2 \Gamma_{v}$, so $x \notin U_{v} K^{* 2}$. Since $\left(1+M_{v}\right) K^{* 2} \subseteq U_{v} K^{* 2}$ this implies $x \notin\left(1+M_{v}\right) K^{* 2}$.

Theorem 5.3. Suppose $K, L$ are fields, $\alpha: Q(K) \rightarrow Q(L)$ is a hyperfield isomorphism and $v$ is a valuation on $K$ such that $\Gamma_{v}$ is finitely generated as an abelian group. Suppose either $(i)$ the basic part of $\left(1+M_{v}\right) K^{* 2}$ is $U_{v} K^{* 2}$ and $\left(1+M_{v}\right) K^{* 2}$ is unexceptional, or (ii) the basic part of $\left(1+M_{v}\right) K^{* 2}$ is $\left(1+M_{v}\right) K^{* 2}$ and $\left(1+M_{v}\right) K^{* 2}$ has index 2 in $U_{v} K^{* 2}$. Then there exists a valuation $w$ on $L$ such that the image of $\left(1+M_{v}\right) K^{* 2} / K^{* 2}$ under $\alpha$ is $\left(1+M_{w}\right) L^{* 2} / L^{* 2}$ and $\left(L^{*}: U_{w} L^{* 2}\right) \geq\left(K^{*}: U_{v} K^{* 2}\right)$. If (i) holds, then the image of $U_{v} K^{* 2} / K^{* 2}$ under $\alpha$ is $U_{w} L^{* 2} / L^{* 2}$.

Proof. Let $r:=\operatorname{rk}_{\mathbb{Q}}\left(\Gamma_{v}\right)$. If $r=0$ then $v$ is the trivial valuation on $K$, and we take $w$ to be the trivial valuation on $L$ in this case. Assume now that $r>0$. Set $T:=\left(1+M_{v}\right) K^{* 2}, S:=\left\{s \in L^{*}: \bar{s}=\alpha(\bar{t})\right.$ for some $\left.t \in T\right\} . U_{v} K^{* 2}$ has index $2^{r}$. In case (i) $T$ is unexceptional and $B(T)=U_{v} K^{* 2}$, so $B(T)$ has index $2^{r}$. In case (ii) $B(T)=T$ has index $2^{r+1}$. The results for $T$ and $B(T)$ carry over to $S$ and $B(S)$ via $\alpha$, i.e., in case (i), $S$ is unexceptional and $B(S)$ is a group of index $2^{r}$ and, in case (ii), $S$ has index $2^{r+1}$ and $B(S)=S$. Applying Theorem 4.5 to the subgroup $S$ of $L^{*}$, there exists a valuation $w$ of $L$ with $\left(1+M_{w}\right) L^{* 2} \subseteq S$, and $U_{w} L^{* 2}$ has index $\geq 2^{r}$. In case (i) we can also assume $U_{w} L^{* 2} \subseteq B(S)$. Let $S^{\prime}=\left(1+M_{w}\right) L^{* 2}$, $T^{\prime}=\left\{t \in K^{*}: \alpha(\bar{t})=\bar{s}\right.$ for some $\left.s \in S^{\prime}\right\}$. Note that $B\left(S^{\prime}\right) \subseteq U_{w} L^{* 2}$ so the group $B\left(S^{\prime}\right)$ has index $\geq 2^{r}$, and, consequently, the group $B\left(T^{\prime}\right)$ has index $\geq 2^{r}$. If $S^{\prime}=S$, 
equivalently, $T^{\prime}=T$, we are done. Suppose now that $T^{\prime} \varsubsetneqq T$ (so, in particular, $T^{\prime}$ has index $\geq 2^{r+2}$ ). Applying Theorem 4.5 one more time, there exists a valuation $v^{\prime}$ of $K$ with $\left(1+M_{v^{\prime}}\right) K^{* 2} \subseteq T^{\prime}$ and $U_{v^{\prime}} K^{* 2}$ has index $\geq 2^{r}$. (If $T^{\prime}$ is unexceptional this is clear. If $T^{\prime}$ is exceptional this is also clear, since then $B\left(T^{\prime}\right)= \pm T^{\prime}$ has index $\geq 2^{r+1}$.) Then $\left(1+M_{v^{\prime}}\right) K^{* 2} \varsubsetneqq\left(1+M_{v}\right) K^{* 2}$. Since $\left(1+M_{v^{\prime}}\right) K^{* 2} \subseteq\left(1+M_{v}\right) K^{* 2}$, $v, v^{\prime}$ are comparable, by Lemma 5.2. Since $\left(1+M_{v}\right) K^{* 2} \nsubseteq\left(1+M_{v^{\prime}}\right) K^{* 2}, v^{\prime} \npreceq v$. Consequently, $v \supsetneqq v^{\prime}$, so $\Gamma_{v^{\prime}}$ is a proper quotient of $\Gamma_{v}$. This contradicts the fact

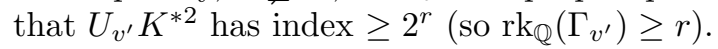

\section{Proposition 5.4.}

(1) Suppose $K, L$ are fields and $\alpha: Q(K) \rightarrow Q(L)$ is a hyperfield isomorphism such that the image of $\left(1+M_{v}\right) K^{* 2} / K^{* 2}$ under $\alpha$ is $\left(1+M_{w}\right) L^{* 2} / L^{* 2}$. Then $\alpha$ induces a hyperfield isomorphism $K / m\left(1+M_{v}\right) K^{* 2} \rightarrow L / m\left(1+M_{w}\right) L^{* 2}$ such that the obvious diagram

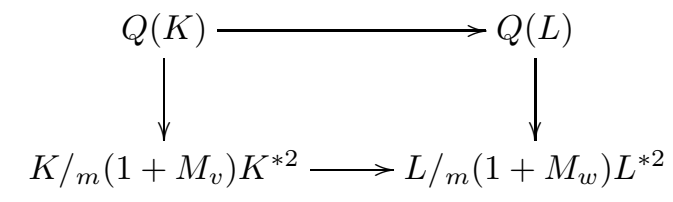

commutes.

(2) If, in addition, the image of $U_{v} K^{* 2} / K^{* 2}$ under $\alpha$ is $U_{w} L^{* 2} / L^{* 2}$, then $\alpha$ induces a hyperfield isomorphism $Q\left(K_{v}\right) \rightarrow Q\left(L_{w}\right)$ and a group isomorphism $\Gamma_{v} / 2 \Gamma_{v} \rightarrow \Gamma_{w} / 2 \Gamma_{w}$ such that the obvious diagrams

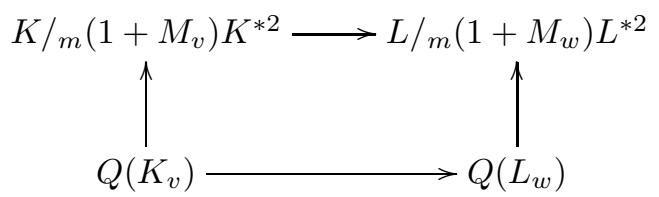

and

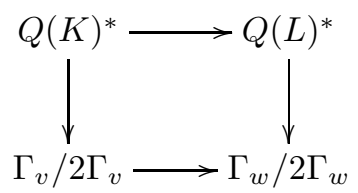

commute. We are assuming here that $v, w$ are non-trivial.

Proof. (1) Since the image of $\left(1+M_{v}\right) K^{* 2} / K^{* 2}$ under $\alpha$ is $\left(1+M_{w}\right) L^{* 2} / L^{* 2}, \alpha$ induces a unique bijection $\bar{\alpha}: K / m\left(1+M_{v}\right) K^{* 2} \rightarrow L / m\left(1+M_{w}\right) L^{* 2}$ such that the diagram (5.1) commutes. Applying Proposition 4.1 (3) one sees that $\bar{\alpha}$ is a hyperfield isomorphism. (2) By our hypothesis the image of $U_{v} K^{* 2} /\left(1+M_{v}\right) K^{* 2}$ under $\bar{\alpha}$ is $U_{w} L^{* 2} /\left(1+M_{w}\right) L^{* 2}$, so $\bar{\alpha}$ induces a bijection $\alpha^{\prime}: Q\left(K_{v}\right) \rightarrow Q\left(L_{w}\right)$ such that the diagram (5.2) commutes. Applying Proposition 4.2 one sees that $\alpha^{\prime}$ is a hyperfield isomorphism. The last assertion is obvious.

\section{Abhyankar VAluations on FUnCtion FiELds OVER GLOBAL FiELDS}

Suppose $K$ and $k$ are fields. We say $K$ is a function field over $k$ if $K$ is a finitely generated field extension of $k$. If $\operatorname{trdeg}(K: k)=n$ we say $K$ is a function field in $n$ variables over $k$. The field of constants of $K$ over $k$ (i.e., the algebraic closure of $k$ 
in $K$ ) is a finite extension of $k$ [28, Chapter 10, Proposition 3]. We do not require that $k$ is the field of constants of $K$ over $k$. If $K$ is a function field over $k$ and $v$ is a valuation on $K$, the Abhyankar inequality asserts that

$$
\operatorname{trdeg}(K: k) \geq \operatorname{rk}_{\mathbb{Q}}\left(\Gamma_{v} / \Gamma_{v \mid k}\right)+\operatorname{trdeg}\left(K_{v}: k_{v \mid k}\right),
$$

where $v \mid k$ denotes the restriction of $v$ to $k$. We will say the valuation $v$ is Abhyankar (relative to $k$ ) if

$$
\operatorname{trdeg}(K: k)=\operatorname{rk}_{\mathbb{Q}}\left(\Gamma_{v} / \Gamma_{v \mid k}\right)+\operatorname{trdeg}\left(K_{v}: k_{v \mid k}\right) .
$$

In this case it is well known that $\Gamma_{v} / \Gamma_{v \mid k}$ is finitely generated and $K_{v}$ is a function field over $k_{v \mid k}$. For a proof of these assertions see [25, Corollary 26].

A global field is a field which is either a number field, i.e., a finite extension of $\mathbb{Q}$, or a function field of transcendence degree 1 over a finite field.

We are interested here in function fields over global fields, equivalently, function fields of transcendence degree $\geq 0$ over $\mathbb{Q}$ or function fields of transcendence degree $\geq 1$ over $\mathbb{F}_{p}$ for some prime $p$. If $K$ is any field we define the nominal transcendence degree of $K$ to be

$$
\operatorname{ntd}(K):=\left\{\begin{array}{ll}
\operatorname{trdeg}(K: \mathbb{Q}) & \text { if } \operatorname{char}(K)=0 \\
\operatorname{trdeg}\left(K: \mathbb{F}_{p}\right)-1 & \text { if } \operatorname{char}(K)=p \neq 0
\end{array} .\right.
$$

Thus, if $K$ is a function field over a global field $k$, then $\operatorname{ntd}(K)=\operatorname{trdeg}(K: k)$. In this situation, for any valuation $v$ of $K$,

and

$$
\operatorname{rk}_{\mathbb{Q}}\left(\Gamma_{v}\right):=\left\{\begin{array}{ll}
\operatorname{rk}_{\mathbb{Q}}\left(\Gamma_{v} / \Gamma_{v \mid k}\right) & \text { if } v \mid k \text { is trivial } \\
\operatorname{rk}_{\mathbb{Q}}\left(\Gamma_{v} / \Gamma_{v \mid k}\right)+1 & \text { if } v \mid k \text { is discrete rank 1 }
\end{array},\right.
$$

$$
\operatorname{ntd}\left(K_{v}\right):=\left\{\begin{array}{ll}
\operatorname{trdeg}\left(K_{v}: k_{v \mid k}\right) & \text { if } v \mid k \text { is trivial } \\
\operatorname{trdeg}\left(K_{v}: k_{v \mid k}\right)-1 & \text { if } v \mid k \text { is discrete rank } 1
\end{array} .\right.
$$

It follows, for any valuation $v$ of $K$, the Abhyankar inequality implies

$$
\operatorname{ntd}(K) \geq \operatorname{rk}_{\mathbb{Q}}\left(\Gamma_{v}\right)+\operatorname{ntd}\left(K_{v}\right)
$$

and $v$ is Abhyankar (relative to $k$ ) iff

$$
\operatorname{ntd}(K)=\operatorname{rk}_{\mathbb{Q}}\left(\Gamma_{v}\right)+\operatorname{ntd}\left(K_{v}\right)
$$

Moreover, if $v$ is Abhyankar (relative to $k$ ) then

$$
\Gamma_{v} \cong \mathbb{Z} \times \cdots \times \mathbb{Z}
$$

(with $\operatorname{rk}_{\mathbb{Q}}\left(\Gamma_{v}\right)$ factors) and $K_{v}$ is either a function field over a global field (if $\operatorname{ntd}\left(K_{v}\right) \geq 0$ ) or a finite field (if $\operatorname{ntd}\left(K_{v}\right)=-1$ ).

\section{WitT EQUIVALENCE OF FUNCTION FIELDS OVER GLOBAL FIELDS}

The main result in this section is Theorem 7.5 which explains how a Witt equivalence of function fields over global fields induces a natural bijection between Abhyankar valuations.

It is important to point out that the bijection between Abhyankar valuations of function fields over global fields is very special. In general, Witt equivalence of two fields does not imply any bijection between valuations whatsoever, as shown in the following simple example: 
Example 7.1. Let $F=k((t))$, where $k$ is an algebraically closed field, char $k \neq 2$. Denote by $v$ the natural valuation on $F$, i.e.,

$$
v\left(\sum_{i=n}^{\infty} a_{i} t^{i}\right):=\min \left\{i: a_{i} \neq 0\right\} \text { if } \sum_{i=n}^{\infty} a_{i} t^{i} \neq 0 .
$$

The residue field of $(F, v)$ is $k$, the value group is $\mathbb{Z}$. Applying Proposition 4.2 we see that $Q(F)$ is a group extension of $Q(k)=\{0,1\}$ by a cyclic group of order 2, so $Q(F)=\{0,1, p\}, p \in Q(F) \backslash Q(k), a+0=a, 1+1=p+p=\{0,1, p\}, 1+p=\{1, p\}$, $a \cdot 0=0,1 \cdot 1=p \cdot p=1,1 \cdot p=p$. It is not difficult to check that exactly the same identities hold true for $Q\left(\mathbb{F}_{5}\right)$, so that $Q(F) \cong Q\left(\mathbb{F}_{5}\right)$ and thus $F \sim \mathbb{F}_{5}$. At the same time, $F$ has lots of non-trivial valuations, whereas $\mathbb{F}_{5}$ has only the trivial one.

We begin with some preliminary results.

Lemma 7.2. Suppose $K$ is a function field over a global field. Then

(1) There are infinitely many discrete rank one Abhyankar valuations $v$ on $K$.

(2) The group $K^{*} / K^{* 2}$ is infinite.

(3) For any $x \in K^{*}, \exists y \in K^{* 2}+x K^{* 2}, y \notin K^{* 2} \cup x K^{* 2}$. If $\operatorname{char}(K) \neq 2$ or $x \notin K^{* 2}$ one can choose $y \neq 0$.

(4) $B\left(K^{* 2}\right)=K^{*}$.

All of this seems to be well-known. Anyway, here is a proof.

Proof. (1) This is clear if $K$ is a number field. Otherwise, $\exists$ a subfield $K_{0} \subseteq K$, $\operatorname{ntd}\left(K_{0}\right)=\operatorname{ntd}(K)-1$. Fix $x \in K$ transcendental over $K_{0} . K$ is a finite extension of $K_{0}(x)$. The principal ideal domain $K_{0}[x]$ has infinitely many irreducibles. Each irreducible $f$ of $K_{0}[x]$ defines a discrete rank one valuation $v_{f}$ on $K_{0}(x)$ with residue field $K_{0}[x] /(f)$. The valuation $v_{f}$ extends in some (possibly nonunique) way to a discrete rank one valuation on $K$ whose residue field is some finite extension of $K_{0}[x] /(f)$. (2) is true for any field $K$ having infinitely many inequivalent discrete rank one valuations. Let $v_{1}, \ldots, v_{n}$ be inequivalent discrete rank one valuations on $K$. Use the approximation theorem to produce $x_{i} \in K^{*}$, $i=1, \ldots, n$ so that $v_{i}\left(x_{j}\right)=\delta_{i j}$ (Kronecker's delta), for $i, j=1, \ldots, n$. Then the $2^{n}$ products $x_{1}^{e_{1}} \ldots x_{n}^{e_{n}}, e_{i} \in\{0,1\}$, belong to distinct square classes. This proves $\left|K^{*} / K^{* 2}\right| \geq 2^{n}$. Since $n$ is can be chosen to be any positive integer, the result follows. (3) Suppose first that $\operatorname{char}(K)=2$. If $x \in K^{* 2}$ one can choose $y=0$. If $x \notin K^{* 2}$ one can choose $y=1+x$. Suppose now that $\operatorname{char}(K) \neq 2$. Let $v$ be a discrete rank one Abhyankar valuation on $K$ with $\operatorname{char}\left(K_{v}\right) \neq 2$. Suppose first that $x \in\left(1+M_{v}\right) K^{* 2}$, say $x=u c^{2}, u \in 1+M_{v}, c \in K^{*}$. By induction on the transcendence degree, there exists $\pi(z) \in K_{v}^{*}$ and $\pi(d), \pi(e) \in K_{v}^{*}$ such that $\pi(z)=\pi(d)^{2}+\pi(e)^{2}, \pi(z) \notin K_{v}^{* 2}$. Take $y=c^{2}\left(d^{2}+u e^{2}\right)=(c d)^{2}+x e^{2}$. Then $y \notin K^{* 2} \cup x K^{* 2}$. If such a valuation $v$ does not exist, then there exist inequivalent discrete rank one valuations $v, w$ on $K$ with $x \notin\left(1+M_{v}\right) K^{* 2}, x \notin\left(1+M_{w}\right) K^{* 2}$. In this case, use the approximation theorem to choose $a \in K^{*}$ so that $v\left(a^{2}\right)>v(x)$, $w\left(a^{2}\right)<w(x)$. Define $y=a^{2}+x$. Then $y=x\left(1+\frac{a^{2}}{x}\right) \in x\left(1+M_{v}\right) K^{* 2}$, so $y \notin K^{* 2}$. Similarly, $y=a^{2}\left(1+\frac{x}{a^{2}}\right) \in\left(1+M_{w}\right) K^{* 2}$, so $y \notin x K^{* 2}$. (4) This is immediate from (3).

Theorem 7.3. Suppose $K$ is a function field over a global field and $v$ is an $A b$ hyankar valuation on $K$. Then: 
(1) $\left(K^{*}: U_{v} K^{* 2}\right)=2^{\mathrm{rk} \Gamma_{Q}\left(\Gamma_{v}\right)}$.

(2) $\left(U_{v} K^{* 2}:\left(1+M_{v}\right) K^{* 2}\right)=\left\{\begin{array}{ll}\infty & \text { if } \operatorname{ntd}\left(K_{v}\right) \geq 0 \\ 2 & \text { if } K_{v} \text { is finite, } \operatorname{char}\left(K_{v}\right) \neq 2 \\ 1 & \text { if } K_{v} \text { is finite, } \operatorname{char}\left(K_{v}\right)=2\end{array}\right.$.

(3) The basic part of $T:=\left(1+M_{v}\right) K^{* 2}$ is

$$
\begin{cases}U_{v} K^{* 2} & \text { if } \operatorname{ntd}\left(K_{v}\right) \geq 0 \\ \pm T=U_{v} K^{* 2} & \text { if } K_{v} \text { is finite, } \operatorname{char}\left(K_{v}\right) \neq 2,-1 \notin K_{v}^{* 2} \\ T & \text { if } K_{v} \text { is finite, } \operatorname{char}\left(K_{v}\right) \neq 2,-1 \in K_{v}^{* 2} \\ T=U_{v} K^{* 2} & \text { if } K_{v} \text { is finite, } \operatorname{char}\left(K_{v}\right)=2\end{cases}
$$

Proof. (1) is immediate from the isomorphism $K^{*} / U_{v} K^{* 2} \cong \Gamma_{v} / 2 \Gamma_{v}$. For (2) and (3) one uses the isomorphism $U_{v} K^{* 2} /\left(1+M_{v}\right) K^{* 2} \cong K_{v}^{*} / K_{v}^{* 2}$ described in Section 4. The assertion in (2) in the case $\operatorname{ntd}\left(K_{v}\right) \geq 0$ follows from Lemma 7.2 (2) applied to the field $K_{v}$. The assertions in (2) in the cases where $K_{v}$ is a finite field are clear. For assertion (3), we apply Proposition 4.6 (2). If $\operatorname{ntd}\left(K_{v}\right) \geq 0$ then $B\left(K_{v}^{* 2}\right)=K_{v}^{*}$, by Lemma 7.2, so $B(T)=U_{v} K^{* 2}$. Suppose now that $K_{v}$ is finite. If $\operatorname{char}\left(K_{v}\right)=2$ then $K_{v}^{*}=K_{v}^{* 2}$ so $B(T)=T=U_{v} K^{* 2}$. If $\operatorname{char}\left(K_{v}\right) \neq 2,-1 \notin K_{v}^{* 2}$, then $B\left(K_{v}^{* 2}\right)= \pm K_{v}^{* 2}=K_{v}^{*}$, so $B(T)= \pm T=U_{v} K^{* 2}$. Finally, if $\operatorname{char}\left(K_{v}\right) \neq 2$, $-1 \in K_{v}^{* 2}$, then $B\left(K_{v}^{* 2}\right)=K_{v}^{* 2}$, so $B(T)=T$.

Lemma 7.4. Suppose $K$ is a function field over a global field, $L$ is a field, and $\alpha: Q(K) \rightarrow Q(L)$ is a hyperfield isomorphism. Then $\operatorname{ntd}(L) \geq \operatorname{ntd}(K)$.

Proof. Let $n:=\operatorname{ntd}(K)$. Pick any Abhyankar valuation $v$ on $K$ with $\operatorname{rk}_{\mathbb{Q}}\left(\Gamma_{v}\right)=n$, i.e., $K_{v}$ is a global field. Choose $w$ as in Theorem 5.3. By Proposition 5.4 $K_{v} \sim L_{w}$. By Lemma $7.2(2), K_{v}^{*} / K_{v}^{* 2} \cong L_{w}^{*} / L_{w}^{* 2}$ is an infinite group, so if $\operatorname{char}\left(L_{w}\right)=p$, $p \neq 0$, then $\operatorname{trdeg}\left(L_{w}: \mathbb{F}_{p}\right) \geq 1$. By Theorem 5.3] $\left|\Gamma_{w} / 2 \Gamma_{w}\right| \geq 2^{n}$, so, by Lemma 5.1. $\left.\operatorname{rk}_{\mathbb{Q}}\left(\Gamma_{w}\right)\right) \geq n$. The result follows from these two facts and the Abhyankar inequality. In more detail, if $\operatorname{char}(L)=p \neq 0$, then $w$ restricted to $\mathbb{F}_{p}$ is trivial and $\operatorname{trdeg}\left(L: \mathbb{F}_{p}\right) \geq \operatorname{rk}_{\mathbb{Q}}\left(\Gamma_{w}\right)+\operatorname{trdeg}\left(L_{w}: \mathbb{F}_{p}\right) \geq n+1$. Similarly, if $\operatorname{char}(L)=0$, then $\operatorname{trdeg}(L: \mathbb{Q}) \geq n+0=n$ or $(n-1)+1=n$, depending on whether $\left.w\right|_{\mathbb{Q}}$ is trivial or $p$-adic.

Theorem 7.5. Suppose $K, L$ are function fields over global fields and $\alpha: Q(K) \rightarrow$ $Q(L)$ is a hyperfield isomorphism. Then:

(1) $\operatorname{ntd}(K)=\operatorname{ntd}(L)$.

(2) For each Abhyankar valuation $v$ of $K$ with $K_{v}$ not finite of characteristic 2 there exists a unique Abhyankar valuation $w$ of $L$ such that $\alpha$ maps $(1+$ $\left.M_{v}\right) K^{* 2} / K^{* 2}$ onto $\left(1+M_{w}\right) L^{* 2} / L^{* 2} . \quad L_{w}$ is also not finite of characteristic 2 , $\operatorname{rk}_{\mathbb{Q}}\left(\Gamma_{v}\right)=\operatorname{rk}_{\mathbb{Q}}\left(\Gamma_{w}\right)$ and $\operatorname{ntd}\left(K_{v}\right)=\operatorname{ntd}\left(L_{w}\right)$.

(3) $\alpha$ maps $U_{v} K^{* 2} / K^{* 2}$ onto $U_{w} L^{* 2} / L^{* 2}$ except possibly when $K_{v}$ is finite, $\operatorname{char}\left(K_{v}\right) \neq 2$ and $-1 \in K_{v}^{* 2}$.

(4) For $v, w$ non-trivial, $\alpha$ induces a hyperfield isomorphism $K / m\left(1+M_{v}\right) K^{* 2} \rightarrow$ $L / m\left(1+M_{w}\right) L^{* 2}$ such that diagram (5.1) commutes. If, in addition, $\alpha$ maps $U_{v} K^{* 2} / K^{* 2}$ onto $U_{w} L^{* 2} / L^{* 2}$ then $\alpha$ induces a hyperfield isomorphism $Q\left(K_{v}\right) \rightarrow$ $Q\left(L_{w}\right)$ and a group isomorphism $\Gamma_{v} / 2 \Gamma_{v} \rightarrow \Gamma_{w} / 2 \Gamma_{w}$ such that diagrams (5.2) and (5.3) commute.

(5) If $v$ corresponds to $w$ and $v^{\prime}$ corresponds to $w^{\prime}$ then $v^{\prime}$ is coarser than $v$ iff $w^{\prime}$ is coarser than $w$. 
Note: One can show that $Q\left(K_{v}\right) \cong Q\left(L_{w}\right)$ as hyperfields, and $\Gamma_{v} / 2 \Gamma_{v} \cong \Gamma_{w} / 2 \Gamma_{w}$ as groups, even in the case where $\alpha$ does not map $U_{v} K^{* 2} / K^{* 2}$ onto $U_{w} L^{* 2} / L^{* 2}$.

Proof. (1) follows from Lemma 7.4 and the symmetry of the hypothesis. (2) Let $n=\operatorname{ntd}(K)=\operatorname{ntd}(L)$. Suppose $w, w^{\prime}$ are Abhyankar valuations on $L$ and $(1+$ $\left.M_{w^{\prime}}\right) L^{* 2}=\left(1+M_{w}\right) L^{* 2}$. If these groups have infinite index, then the basic parts of these groups are the same, i.e., $U_{w^{\prime}} L^{* 2}=U_{w} L^{* 2}$, i.e., $\operatorname{rk}_{\mathbb{Q}}\left(\Gamma_{w^{\prime}}\right)=\operatorname{rk}_{\mathbb{Q}}\left(\Gamma_{w}\right)$, by Theorem 7.3. If these groups have finite index then $\operatorname{rk}_{\mathbb{Q}}\left(\Gamma_{w^{\prime}}\right)=n+1=\operatorname{rk}_{\mathbb{Q}}\left(\Gamma_{w}\right)$, again by Theorem 7.3 . Since we already know, by Lemma 5.2, that $w$ and $w^{\prime}$ are comparable, this proves $w^{\prime}=w$. This proves the uniqueness of $w$. Suppose now that $v$ is an Abhyankar valuation of $K, K_{v}$ not finite of characteristic 2 . The valuation $w$ exists by Theorem 5.3. Let $r=\operatorname{rk}_{\mathbb{Q}}\left(\Gamma_{v}\right), s=\operatorname{ntd}\left(K_{v}\right)$. If $s \geq 0$ then $\alpha$ induces an isomorphism $Q\left(K_{v}\right) \rightarrow Q\left(L_{w}\right)$, by Proposition 5.4. so $\operatorname{ntd}\left(L_{w}\right) \geq s$, by Lemma 7.4. If $s=-1$ then $\operatorname{ntd}\left(L_{w}\right) \geq s$ holds trivially. Also, $\left|\Gamma_{w} / 2 \Gamma_{w}\right| \geq\left|\Gamma_{v} / 2 \Gamma_{v}\right|=2^{r}$, so $\operatorname{rk}_{\mathbb{Q}}\left(\Gamma_{w}\right) \geq r$. Thus

$$
\operatorname{ntd}(L) \geq \operatorname{rk}_{\mathbb{Q}}\left(\Gamma_{w}\right)+\operatorname{ntd}\left(L_{w}\right) \geq r+s=\operatorname{ntd}(K)=\operatorname{ntd}(L),
$$

so $w$ is Abhyankar, $\operatorname{rk}_{\mathbb{Q}}\left(\Gamma_{w}\right)=r$, and $\operatorname{ntd}\left(L_{w}\right)=s$. In particular, $\left(U_{w} L^{* 2}\right.$ : $\left.\left(1+M_{w}\right) L^{* 2}\right) \geq 2$, so $L_{w}$ is not finite of characteristic 2 . This proves (2). (3) and (4) are straightforward. (5) Suppose now that $v \leftrightarrow w, v^{\prime} \leftrightarrow w^{\prime}, v \preceq v^{\prime}$. Then $\left(1+M_{v^{\prime}}\right) K^{* 2} \subseteq\left(1+M_{v}\right) K^{* 2}$, so $\left(1+M_{w^{\prime}}\right) L^{* 2} \subseteq\left(1+M_{w}\right) L^{* 2}$. By Lemma 5.2. $w$ and $w^{\prime}$ are comparable. If $w^{\prime} \preceq w$ then $\left(1+M_{w}\right) L^{* 2} \subseteq\left(1+M_{w^{\prime}}\right) L^{* 2}$ so $\left(1+M_{w^{\prime}}\right) L^{* 2}=\left(1+M_{w}\right) L^{* 2}$. We already know that $w=w^{\prime}$ holds in this case. Thus $w \preceq w^{\prime}$ holds in any case. This proves (5).

The next two lemmas allow one to distinguish the characteristic 2 case from the characteristic $\neq 2$ case. Denote by $\bar{t} \in K^{*} / K^{* 2}$ the image of $t \in K^{*}$.

Lemma 7.6. Suppose $K$ is a field, $\operatorname{char}(K)=2, \bar{x}, \bar{y} \in K^{*} / K^{* 2}, \bar{x}, \bar{y} \neq 1$ and $\bar{y} \in D_{K}\langle 1, \bar{x}\rangle$. Then $D_{K}\langle 1, \bar{y}\rangle=D_{K}\langle 1, \bar{x}\rangle$.

Proof. Suppose $\bar{z} \in D_{K}\langle 1, \bar{y}\rangle$. By our assumptions, $x, y, z \in K^{*}, x, y \notin K^{* 2}$, $y=a^{2}+b^{2} x, z=c^{2}+d^{2} y, a, b, c, d \in K$. It follows that $z=c^{2}+d^{2}\left(a^{2}+b^{2} x\right)=$ $(c+a d)^{2}+(b d)^{2} x$, so $\bar{z} \in D_{K}\langle 1, \bar{x}\rangle$. This proves the inclusion $D_{K}\langle 1, \bar{y}\rangle \subseteq D_{K}\langle 1, \bar{x}\rangle$. The other inclusion follows from this one, using the symmetry of the hypothesis (i.e., using $\bar{y} \in D_{K}\langle 1, \bar{x}\rangle \Leftrightarrow \bar{x} \in D_{K}\langle 1, \bar{y}\rangle$ ).

Lemma 7.7. Suppose $K$ is a function field over a global field, $\operatorname{char}(K) \neq 2$. Then there exists $\bar{x}, \bar{y} \in K^{*} / K^{* 2}, \bar{x}, \bar{y} \neq 1$ such that $\bar{y} \in D_{K}\langle 1, \bar{x}\rangle, D_{K}\langle 1, \bar{y}\rangle \nsubseteq D_{K}\langle 1, \bar{x}\rangle$.

Proof. Fix inequivalent discrete rank one Abhyankar valuations $v, w$ on $K$ with $\operatorname{char}\left(K_{v}\right), \operatorname{char}\left(K_{w}\right) \neq 2$. Choose $x$ so that $v(x)=w(x)=1$ and $a_{0}, b_{0}$ so that $w\left(a_{0}\right)=w\left(b_{0}\right)=0$ and the image of $c=a_{0}^{2}+b_{0}^{2}$ in the residue field of $w$ is not a square. This is possible by Lemma 7.2 (3). Define $y=a^{2}+x, z=b^{2}+y$ (so $\left.z=a^{2}+b^{2}+x\right)$ where $a, b$ are such that $v(a)>0, w\left(a-a_{0}\right)>0, w\left(b-b_{0}\right)>0$. Then $v(y)=v(x)=1$, so $x, y \notin K^{* 2}$ and $w\left(a^{2}+b^{2}-c\right)>0$ so $z=a^{2}+b^{2}+x \in c\left(1+M_{w}\right)$. Thus $x, y, z \in K^{*}, \bar{y} \in D_{K}\langle 1, \bar{x}\rangle, \bar{z} \in D_{K}\langle 1, \bar{y}\rangle, \bar{x} \neq 1, \bar{y} \neq 1$. Let $T=\left(1+M_{w}\right) K^{* 2}$. Thus $T+x T=T \cup x T$ and $z \notin T \cup x T$, so $\bar{z} \notin D_{K}\langle 1, \bar{x}\rangle$.

Corollary 7.8. Let $K, L$ be function fields over global fields and $K \sim L$. Then

(1) $\operatorname{char}(K)=0$ iff $\operatorname{char}(L)=0$,

(2) $\operatorname{char}(K)=2$ iff $\operatorname{char}(L)=2$. 
Proof. For (1), assume $\operatorname{char}(K)=0$. Fix an Abhyankar valuation $v$ of $K$ such that $K_{v}$ is a number field (so $K_{v}$ possesses a dyadic valuation). Denote by $w$ the corresponding Abhyankar valuation on $L$. Thus $L_{w}$ is a global field and $K_{v} \sim L_{w}$. Applying [42, Theorem 1.1], $L_{w}$ also possesses a dyadic valuation so is also a number field. This proves $\operatorname{char}(L)=0$. (2) Assume $\operatorname{char}(K)=2$. Applying Lemma 7.6 and Lemma 7.7, we see that $\operatorname{char}(L)=2$.

Remark 7.9. (1) For a global field $K$ the square of the fundamental ideal of its Witt ring of non-singular symmetric bilinear forms vanishes, if $K$ has characteristic 2 ([33, Theorem III.5.10]) and does not vanish for global fields of any other characteristic (see [33, Chapter III]). Hence, if $K$ and $L$ are Witt equivalent global fields and one field has characteristic 2, the other does also. Corollary 7.8 can be viewed as a certain generalization of this observation.

(2) Any two quadratically closed fields are Witt equivalent, regardless of their characteristics, their Witt ring being just $\mathbb{Z} / 2 \mathbb{Z}$ ([27, Proposition 3.1], 33, Remark III.3.4]). Therefore it is, in principle, possible to provide an example of two Witt equivalent fields $K$ and $L$ with $\operatorname{char} K=2$ and $\operatorname{char} L \neq 2$. However, the authors are not aware of any other examples.

Lemma 7.10. If $K$ is a function field over a field $k$, $\operatorname{char}(k)=2$, then

$$
\left[K: K^{2}\right]=2^{\operatorname{trdeg}(K: k)} \cdot\left[k: k^{2}\right] .
$$

Proof. Let $n:=\operatorname{trdeg}(K: k)$. Fix $x_{1}, \ldots, x_{n}$ in $K$ algebraically independent over $k$. Then $K$ is a finite extension of $k\left(x_{1}, \ldots, x_{n}\right)$. The map $a \mapsto a^{2}$ defines an isomorphism from $K$ onto $K^{2}$ which maps $k\left(x_{1}, \ldots, x_{n}\right)$ onto $k^{2}\left(x_{1}^{2}, \ldots, x_{n}^{2}\right)$. It follows that $\left[K^{2}: k^{2}\left(x_{1}^{2}, \ldots, x_{n}^{2}\right)\right]=\left[K: k\left(x_{1}, \ldots, x_{n}\right)\right]$. Thus we are reduced to showing that $\left[k\left(x_{1}, \ldots, x_{n}\right): k^{2}\left(x_{1}^{2}, \ldots, x_{n}^{2}\right)\right]=2^{n}\left[k: k^{2}\right]$. But this is clear.

\section{Remark 7.11.}

(1) It follows from results in 4 (specifically, from 4, Theorem 2.9 and Proposition 2.10]) that (i) if $K, L$ are global fields of characteristic 2 then $K \sim L$, and (ii) if $K, L$ are function fields over global fields of characteristic 2 of nominal transcendence degree 1 or more then $K \sim L$ iff $K \cong L$. One obtains these results by applying Lemma 7.10 , taking $k=\mathbb{F}_{2}$.

(2) For $K, L$ global fields of characteristic $\neq 2$ the meaning of $K \sim L$ is well understood; see for example [5, Theorem 3.1 and Corollary 3.2].

The relationship between Abhyankar valuations $v$ on $K$ with $K_{v}$ finite, $\operatorname{char}\left(K_{v}\right)=$ 2 and Abhyankar valuations $w$ on $L$ with $L_{v}$ finite, $\operatorname{char}\left(L_{w}\right)=2$ seems to be not very well understood.

\section{Remark 7.12.}

(1) If $K$ and $L$ are number fields and $\alpha: Q(K) \rightarrow Q(L)$ is a hyperfield isomorphism the arguments in 42 show that for each dyadic valuation $v$ of $K$ there exists a unique dyadic valuation $w$ of $L$ such that $\alpha$ maps $\left(1+4 M_{v}\right) K^{* 2} / K^{* 2}$ onto $\left(1+4 M_{w}\right) L^{* 2} / L^{* 2}$.

(2) Suppose $v$ is a dyadic valuation on a number field $K$. Denote by $\tilde{K}_{v}$ the completion of $K$ at $v$. The natural embedding $K \hookrightarrow \tilde{K}_{v}$ induces a hyperfield isomorphism $K /{ }_{m} T \cong Q\left(\tilde{K}_{v}\right)$, where $T:=\left(1+4 M_{v}\right) K^{* 2}$. The structure of $Q\left(\tilde{K}_{v}\right)$ is described in [29, Section 3.6] for example. 
(3) Suppose $K$ is a function field over $\mathbb{Q}$ and $v^{\prime}$ is an Abhyankar valuation on $K$ such that the residue field $K_{v^{\prime}}$ is a number field. Suppose also that $v$ is a valuation of $K$ such that $v \preceq v^{\prime}$ and the induced valuation $\bar{v}$ on $K_{v^{\prime}}$ is dyadic. Then $M_{v^{\prime}} \subseteq M_{v}$ and $4 M_{v^{\prime}}=M_{v^{\prime}}\left(\right.$ so $\left.1+M_{v^{\prime}} \subseteq 1+4 M_{v}\right), K / m\left(1+4 M_{v}\right) K^{* 2}$ is a group extension of the hyperfield $K_{v^{\prime}} / m\left(1+4 M_{\bar{v}}\right) K_{v^{\prime}}^{* 2}$ in a natural way, and the following diagram of hyperfields and hyperfield morphisms is commutative:

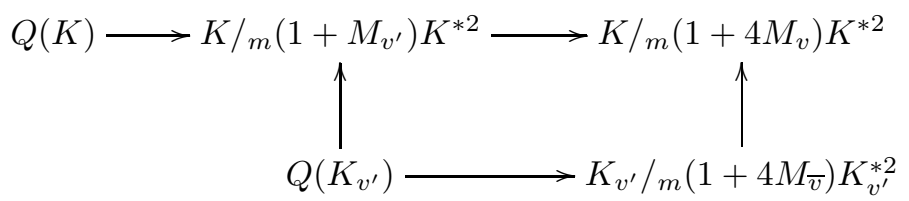

Here, the horizontal arrows are quotient morphisms and the vertical arrows are group extensions.

(4) It follows from (1), (2) and (3) that if $K, L$ are function fields over global fields and $\alpha: Q(K) \rightarrow Q(L)$ is a hyperfield isomorphism, then there is a well-defined bijection $v \leftrightarrow w$ such that $\alpha$ maps $\left(1+4 M_{v}\right) K^{* 2} / K^{* 2}$ onto $\left(1+4 M_{w}\right) L^{* 2} / L^{* 2}$ between Abhyankar valuations $v$ of $K$ with $K_{v}$ finite, $\operatorname{char}\left(K_{v}\right)=2$ such that there exists an Abhyankar valuation $v^{\prime}$ with $v \preceq v^{\prime}$ and $K_{v^{\prime}}$ is a number field and Abhyankar valuations $w$ of $L$ with $L_{w}$ finite, $\operatorname{char}\left(L_{w}\right)=2$ such that there exists an Abhyankar valuation $w^{\prime}$ with $w \preceq w^{\prime}$ and $L_{w^{\prime}}$ is a number field. The proof is omitted.

The relationship between non-Abhyankar valuations $v$ on $K$ and non-Abhyankar valuations $w$ on $L$ is not very well understood. It is known, by results in [25], that the Abhyankar valuations are dense in the spectral space consisting of all valuations, but this does not seem to help very much.

\section{Further APPLiCATIONS}

Let $K$ be a function field in $n$ variables over a global field. For $0 \leq i \leq n$ denote by $\nu_{K, i}$ the set of Abyankar valuations $v$ on $K$ with $\operatorname{ntd}\left(K_{v}\right)=i$. Observe that

$$
\nu_{K, i}=\nu_{K, i, 0} \cup \nu_{K, i, 1} \cup \nu_{K, i, 2} \text { (disjoint union) }
$$

where

$$
\nu_{K, i, j}:=\left\{\begin{array}{l}
\left\{v \in \nu_{K, i}: \operatorname{char}\left(K_{v}\right)=0\right\} \text { if } j=0 \\
\left\{v \in \nu_{K, i}: \operatorname{char}\left(K_{v}\right) \neq 0,2\right\} \text { if } j=1 \\
\left\{v \in \nu_{K, i}: \operatorname{char}\left(K_{v}\right)=2\right\} \text { if } j=2
\end{array} .\right.
$$

Of course, some of the sets $\nu_{K, i, j}$ may be empty. Specifically, if char $(K)=p$ for some odd prime $p$ then $\nu_{K, i, j}=\emptyset$ for $j \in\{0,2\}$, and if $\operatorname{char}(K)=2$ then $\nu_{K, i, j}=\emptyset$ for $j \in\{0,1\}$.

Corollary 8.1. Suppose $K, L$ are function fields in $n$ variables over global fields which are Witt equivalent via a hyperfield isomorphism $\alpha: Q(K) \rightarrow Q(L)$. Then for each $i \in\{0,1, \ldots, n\}$ and each $j \in\{0,1,2\}$ there is a uniquely defined bijection between $\nu_{K, i, j}$ and $\nu_{L, i, j}$ such that, if $v \leftrightarrow w$ under this bijection, then $\alpha$ maps $\left(1+M_{v}\right) K^{* 2} / K^{* 2}$ onto $\left(1+M_{w}\right) L^{* 2} / L^{* 2}$ and $U_{v} K^{* 2} / K^{* 2}$ onto $U_{w} L^{* 2} / L^{* 2}$.

Proof. The correspondence $v \leftrightarrow w$ is the one defined in Theorem 7.5, If $v \leftrightarrow w$ then $K_{v} \sim L_{w}$ so $v \in \nu_{K, i, j} \Leftrightarrow w \in \nu_{L, i, j}$, for each $i$ and $j$. 
Corollary 8.2. Let $K \sim L$ be function fields over number fields, with fields of constants $k$ and $\ell$ respectively. If there exists $v \in \nu_{K, 0,0}$ with $K_{v}=k$ and $w \in \nu_{L, 0,0}$ with $L_{w}=\ell$ then $k \sim \ell$.

Proof. Let $v \leftrightarrow w$ be the bijection between $\nu_{K, 0,0}$ and $\nu_{L, 0,0}$ defined by Corollary 8.1. We know that $K_{v} \sim L_{w}$ for any $v, w$ related in this way. Since $K_{v}$ and $L_{w}$ are number fields, this implies $\left[K_{v}: \mathbb{Q}\right]=\left[L_{w}: \mathbb{Q}\right]$ for any such $v, w$ [42, Proposition 1.5]. We know also that $k \subseteq K_{v}$ and $\ell \subseteq L_{w}$. Choosing $v \leftrightarrow w$ so that $\left[K_{v}: \mathbb{Q}\right]=\left[L_{w}: \mathbb{Q}\right]$ is minimal, we see that $K_{v}=k$ and $L_{w}=\ell$.

\section{Remark 8.3.}

(1) Suppose $K$ is the function field of an irreducible $k$-variety which has a nonsingular $k$-rational point. (This is always the case, for example, if $K$ is purely transcendental over $k$.) Then there exists $v \in \nu_{K, 0,0}$ with $K_{v}=k$. To prove this one uses the fact that if $A$ is a regular local ring of dimension $n$ with maximal ideal $\mathfrak{m}=\left(x_{1}, \ldots, x_{n}\right)$ and residue field $k$, then $A /\left(x_{n}\right)$ is a regular local ring of dimension $n-1$, and the localization of $A$ at the prime ideal $\left(x_{n}\right)$ is a discrete valuation ring with residue field equal to the field of quotients of $A /\left(x_{n}\right)$; e.g., see 3. Chapter 11]. Iterating this procedure yields a chain of Abhyankar valuations $v_{1} \succeq \cdots \succeq v_{n}$ on $K$ with $\operatorname{trdeg}\left(K_{v_{i}}: k\right)=n-i, i=1, \ldots, n$ and $K_{v_{n}}=k$.

(2) If $K$ and $L$ are function fields over global fields of characteristic $\neq 0$, with fields of constants $k$ and $\ell$, respectively, then $K \sim L \Rightarrow k \sim \ell$. If $k, \ell$ have characteristic 2 then $\left[k: k^{2}\right]=\left[\ell: \ell^{2}\right]=2$, by Lemma 7.10, so $k \sim \ell$, by [4, Proposition 2.10]. Suppose $k, \ell$ each have characteristic different from 0 and 2 . Then $k, \ell$ each have level 1 or 2 . If $k$ has level 1 then $K$ and consequently also $L$ has level 1 . Since $\ell$ is algebraically closed in $L$ this implies $\ell$ has level 1 . This proves $k$ and $\ell$ have the same level, so $k \sim \ell$, by [5, Corollary 3.2].

(3) Combining Corollary 8.2 with (1) and (2) we see that, in particular, 21, Proposition 3.2] is indeed true (even though the proof of [21, Proposition 3.2] given in [21] is based on the erroneous argument in [21, Theorem 1.3]).

Suppose now that $k$ is a number field. Then every ordering of $k$ is archimedean, i.e., corresponds to a real embedding $k \hookrightarrow \mathbb{R}$. Let $r_{1}$, respectively $r_{2}$ be the number of real embeddings of $k$, respectively the number of conjugate pairs of complex embeddings of $k$. Thus $[k: \mathbb{Q}]=r_{1}+2 r_{2}$. Let

$$
V_{k}:=\left\{r \in k^{*}:(r)=\mathfrak{a}^{2} \text { for some fractional ideal } \mathfrak{a} \text { of } k\right\} .
$$

Here, $(r)$ denotes the fractional ideal of $k$ generated by $r$. Clearly $V_{k}$ is a subgroup of $k^{*}$ and $k^{* 2} \subseteq V_{k}$.

Lemma 8.4. The 2-rank of $V_{k} / k^{* 2}$ is $r_{1}+r_{2}+2-\operatorname{rk}\left(C_{k}\right)$, where $C_{k}$ denotes the ideal class group of $k$.

Proof. See [8, Lemma 2.4(a)].

Lemma 8.5. Suppose $K=k\left(x_{1}, \ldots, x_{n}\right)$ and $v$ is a discrete rank 1 valuation on $k$. There exists an Abhyankar extension $v^{\prime}$ of $v$ to $K$ such that $\Gamma_{v^{\prime}}=\Gamma_{v}$.

Proof. Define $v^{\prime}$ by

$$
v^{\prime}\left(\sum_{\alpha} a_{\alpha} x^{\alpha}\right):=\min \left\{v\left(a_{\alpha}\right): \alpha \in \mathbb{N}^{n}\right\} \text { and } v^{\prime}\left(\frac{f}{g}\right):=v^{\prime}(f)-v^{\prime}(g) .
$$

Here, $x^{\alpha}:=x_{1}^{\alpha_{1}} \ldots x_{n}^{\alpha_{n}}$, for $\alpha \in \mathbb{N}^{n}$. 
Theorem 8.6. Suppose $K=k\left(x_{1}, \ldots, x_{n}\right)$ and $L=\ell\left(x_{1}, \ldots, x_{n}\right)$ where $n \geq 1$ and $k$ and $\ell$ are number fields, and $\alpha: Q(K) \rightarrow Q(L)$ is a hyperfield isomorphism. Then

(1) $r \in k^{*} / k^{* 2}$ iff $\alpha(r) \in \ell^{*} / \ell^{* 2}$.

(2) The map $r \mapsto \alpha(r)$ defines a hyperfield isomorphism between $Q(k)$ and $Q(\ell)$.

(3) $\alpha$ maps $V_{k} / k^{* 2}$ to $V_{\ell} / \ell^{* 2}$.

(4) The 2-ranks of the ideal class groups of $k$ and $\ell$ are equal.

Proof. Since $k$ is the field of constants of $K$, the canonical group homomorphism from $k^{*} / k^{* 2}$ to $K^{*} / K^{* 2}$ is injective. Claim: The image of the embedding $k^{*} / k^{* 2} \hookrightarrow$ $K^{*} / K^{* 2}$ is equal to $\cap_{v \in \nu_{K, n-1,0}} U_{v} K^{* 2} / K^{* 2}$. One inclusion is clear. For the other, use the fact that $D:=k\left[x_{1}, \ldots, x_{n}\right]$ is a UFD. Suppose $f \in \cap_{v \in \nu_{K, n-1,0}} U_{v} K^{* 2} / K^{* 2}$, $f=\frac{g}{h}, g, h \in D, g, h \neq 0$. Then $f=u p_{1}^{e_{1}} \ldots p_{s}^{e_{s}}, u \in k^{*}, p_{1}, \ldots, p_{s}$ irreducibles in $D$. Consider the discrete rank 1 valuation $v_{i}$ on $K$ associated to $p_{i}$. Then $v_{i} \in \nu_{K, n-1,0}$, so $e_{i}=v_{i}(f)$ is even, $i=1, \ldots, s$. It follows that $p_{1}^{e_{1}} \ldots p_{s}^{e_{s}} \in K^{* 2}$ so $f \equiv u \bmod K^{* 2}$. This proves the claim. Since the image of $\cap_{v \in \nu_{K, n-1,0}} U_{v} K^{* 2} / K^{* 2}$ under $\alpha$ is $\cap_{w \in \nu_{L, n-1,0}} U_{w} L^{* 2} / L^{* 2}$, by Corollary 8.1, this proves (1). Observe that if $v \leftrightarrow w, v \in \nu_{K, 0,0}, w \in \nu_{L, 0,0}$, the diagram

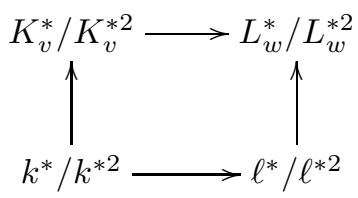

is commutative. The vertical arrows are the maps induced by the field embeddings $k \hookrightarrow K_{v}, \ell \hookrightarrow L_{w}$. Since the top arrow in diagram (8.1) defines a hyperfield isomorphism between $Q\left(K_{v}\right)$ and $Q\left(L_{w}\right)$ we know that $\left[K_{v}: \mathbb{Q}\right]=\left[L_{w}: \mathbb{Q}\right]$. Choose $v, w$ with $\left[K_{v}: \mathbb{Q}\right]=\left[L_{w}: \mathbb{Q}\right]$ minimal. The argument in Corollary 8.2 shows that $K_{v}=k$ and $L_{w}=\ell$. This proves (2). Lemma 8.5 implies that

$$
V_{k} / k^{* 2}=\left\{r \in k^{*} / k^{* 2}: r \in U_{v} K^{* 2} / K^{* 2} \forall v \in \nu_{K, n-1,1} \cup \nu_{K, n-1,2}\right\},
$$

so (3) is clear. Since it is well-known that $r_{1}$ and $r_{2}$ are invariant under Witt equivalence, assertion (4) is immediate now, from (3) and Lemma 8.4. This completes the proof.

Remark 8.7. (1) The fact that Witt rings of number fields carry some data on the parity of class numbers was first noticed in 43, and then some additional results were given in [18. A deeper study of the relations between Witt equivalence of number fields and 2-ranks of ideal class groups can be found in [7.

(2) One can extend Theorem 8.6 a bit: Let $V_{k}^{1}$ denote the set of all $r \in k^{*}$ such that $v(r)$ is even for all non-dyadic valuations $v$ of $k$. By Lemma 8.5,

$$
V_{k}^{1} / k^{* 2}=\left\{r \in k^{*} / k^{* 2}: r \in U_{v} K^{* 2} / K^{* 2} \forall v \in \nu_{K, n-1,1}\right\}
$$

so $\alpha$ maps $V_{k}^{1} / k^{* 2}$ to $V_{\ell}^{1} / \ell^{* 2}$. Applying this in conjunction with the generalization of Lemma 8.4 given in [6. Lemma 2.4] or [45, Proposition 1], we see that the $S$-class groups of $k$ and $\ell$ have the same 2-rank, where $S$ consists of all primes which are infinite or dyadic.

\section{Questions:}

(1) In Theorem 8.6, is the hypothesis that $K$ and $L$ are purely transcendental over $k$ and $\ell$ really necessary? 
(2) For arbitrary fields $K$ and $L$ is it true that $K(x) \sim L(x) \Rightarrow K \sim L$ ?

(3) For fixed integers $n \geq 1, m \geq 2$, are there infinitely many Witt inequivalent fields $k\left(x_{1}, \ldots, x_{n}\right), k$ a number field, $[k: \mathbb{Q}]=m$ ?

Question 3 is interesting because, for given $m$, there are only finitely many Witt inequivalent number fields $k$ with $[k: \mathbb{Q}]=m$. For $m=1,2,3$ and 4 these numbers are $1,7,8$ and 29 respectively; see 7 and 18 .

It is proved in [45] that if $\ell$ is a number field, $[\ell: \mathbb{Q}]$ even, and $\ell \neq \mathbb{Q}(\sqrt{-1})$, then, for each integer $t \geq 1$, there exists a number field $k$ such that $k \sim \ell$ and the 2-rank of the class group of $k$ is $\geq t$. This extends an earlier result in [7].

Corollary 8.8. For fixed $n \geq 1$ and fixed number field $\ell,[\ell: \mathbb{Q}]$ even, $\ell \neq \mathbb{Q}(\sqrt{-1})$, there are infinitely many Witt inequivalent fields of the form $k\left(x_{1}, \ldots, x_{n}\right), k$ a number field, $k \sim \ell$.

For odd degree extensions Question 3 remains open. Table 2 in [45] shows that each of the 8 Witt equivalence classes of cubic extensions contains fields with 2rank of the class group equal to 0,1 , and 2. Results in [11] [38] [39] [47] show that $0,1,2,3,4,5,7$ can occur as the 2 -rank of the class group of a cubic field.

\section{REFERENCES}

[1] J.K. Arason, R. Elman, W. Jacob, Rigid elements, valuations, and realization of Witt rings. J. Algebra 110 (1987) 449-467.

[2] J.K. Arason, A. Pfister, Beweis des Krullschen Durchschnittsatzes für den Wittring. Invent. Math. 12 (1971), 173-176.

[3] M.F Atiyah, I.G. Macdonald, Introduction to commutative algebra. Addison-Wesley Publishing Co., Reading, Mass.-London-Don Mills, Ont. 1969.

[4] R. Baeza, R. Moresi, On the Witt-equivalence of fields of characteristic 2. J. Algebra 92 (1985), no. 2, 446-453.

[5] J. Carpenter, Finiteness theorems for forms over global fields. Math. Z. 209 no. 1 (1992) 153-166.

[6] P.E. Conner, J. Hurrelbrink, The 4-rank of $K_{2}(O)$. Canad. J. Math. 41 (1989), no. 5, 932-960.

[7] P.E. Conner, R. Perlis, K. Szymiczek, Wild sets and 2-ranks of class groups. Acta Arith. 79 (1997), no. 1, 83-91.

[8] A. Czogała, On reciprocity equivalence of quadratic number fields. Acta Arith. 58 (1991), no. $1,27-46$.

[9] M. Dickmann, F. Miraglia, Special groups: Boolean-theoretic methods in the theory of quadratic forms, Mem. Amer. Math. Soc. 145, American Mathematical Society, Providence, RI, 2000.

[10] I. Efrat, Valuations, orderings, and Milnor K-theory. Mathematical Surveys and Monographs, 124. American Mathematical Society, Providence, RI, 2006.

[11] H. Eisenbeis, G. Frey, B. Ommerborn. Computation of the 2-rank of pure cubic fields. Math. Comp. 32 (1978), no. 142, 559-569.

[12] A.J. Engler, A. Prestel, Valued fields. Springer Monographs in Mathematics. Springer-Verlag, Berlin, 2005.

[13] N. Grenier-Boley, D.W. Hoffmann, Isomorphism criteria for Witt rings of real fields. With appendix by Claus Scheiderer. Forum Math. 25 (2013) 1-18.

[14] D.K. Harrison, Witt rings. University of Kentucky Notes, Lexington, Kentucky (1970).

[15] W. Jacob, On the structure of Pythagorean fields. J. Algebra 68 (1981), no. 2, 247-267.

[16] W. Jacob, Quadratic forms over dyadic valued fields. I. The graded Witt ring. Pacific J. Math. 126 no. 1, (1987), 21-79.

[17] W. Jacob, Quadratic forms over dyadic valued fields. II. Relative rigidity and Galois cohomology. J. Algebra 148 (1992), no. 1, 162-202.

[18] S. Jakubec, F. Marko, K. Szymiczek, Parity of class numbers and Witt equivalence of quartic fields. Math. Comp. 64 (1995), no. 212, 1711-1715. 
[19] J.L. Kleinstein, A. Rosenberg, Succinct and representational Witt rings. Pacific J. Math. 86 (1980) 99-137.

[20] M. Knebusch, A. Rosenburg, R. Ware, Structure of Witt rings and quotients of Abelian group rings. Amer. J. Math. 94 (1972), 119-155.

[21] P. Koprowski, Local-global principle for Witt equivalence of function fields over global fields. Colloq. Math. 91 (2002) 293-302.

[22] P. Koprowski, Witt equivalence of algebraic function fields over real closed fields. Math. Z. 242 (2002) 323-345.

[23] M. Krasner, Approximation des corps valués complets de caractéristique $p \neq 0$ par ceux de caractéristique 0. 1957 Colloque d'algèbre supérieure, tenu à Bruxelles du 19 au 22 décembre 1956 pp. 129-206 Centre Belge de Recherches Mathématiques Établissements Ceuterick, Louvain; Librairie Gauthier-Villars, Paris.

[24] M. Krasner, A class of hyperrings and hyperfields, Internat. J. Math. and Math. Sci. 6 (1983) 307-312.

[25] F.-V. Kuhlmann, On places of algebraic function fields in arbitrary characteristic. Advances in Math. 188 (2004) 399-424.

[26] M. Kula, L. Szczepanik, K. Szymiczek, Quadratic form schemes and quaternionic schemes. Fund. Math. 130 (1988), no. 3, 181-190.

[27] T.-Y. Lam, Introduction to quadratic forms over fields. Graduate Studies in Mathematics 67 American Mathematical Society, Providence, RI, 2005.

[28] S. Lang, Algebra. Addison-Wesley Publishing Co., Reading, Mass. 1965

[29] M. Marshall, Abstract Witt rings, Queen's Papers in Pure and Applied Math. 57, Queen's University, Kingston, Ontario (1980).

[30] M. Marshall, The elementary type conjecture in quadratic form theory, Cont. Math $\mathbf{3 4 4}$ (2004), 275-293.

[31] M. Marshall, Real reduced multirings and multifields, J. Pure and Appl. Alg. 205 (2006) $452-468$.

[32] Ch.G. Massouros, Methods of constructing hyperfields. Internat. J. Math. Math. Sci. 8 no. 4 (1985) 725-728.

[33] J. Milnor, D. Husemoller, Symmetric bilinear forms. Ergebnisse der Mathematik und ihrer Grenzgebiete, Band 73. Springer-Verlag, New York-Heidelberg, 1973.

[34] J. Mináč, M. Spira, Witt rings and Galois groups. Ann. of Math. (2) 144 (1996), no. 1, 35-60.

[35] J. Mittas, Sur une classe d'hypergroupes commutatifs. C. R. Acad. Sci. Paris Sér. A-B 269 1969 A485-A488.

[36] R. Perlis, K. Szymiczek, P.E. Conner, R. Litherland, Matching Witts with global fields. Contemp. Math. 155 (1994) 365-378.

[37] P. Ribenboim, Théorie des valuations. Deuxième édition multigraphié. Séminaire de Mathématiques Supérieures, No. 9 (Été, 1964) Les Presses de l’Université de Montréal, Montreal, Que. 1968.

[38] U. Schneiders, Estimating the 2-rank of cubic fields by Selmer groups of elliptic curves. J. Number Theory 62 (1997), no. 2, 375-396.

[39] D. Shanks, The simplest cubic fields. Math. Comp. 28 (1974), 1137-1152.

[40] T.A. Springer, Quadratic forms over fields with a discrete valuation. I. Equivalence classes of definite forms. Indag. Math. 17, (1955) 352-362.

[41] T.A. Springer, Quadratic forms over fields with a discrete valuation. II. Indag. Math. 18 (1956) 238-246.

[42] K. Szymiczek, Matching Witts locally and globally. Math. Slovakia 41 (1991) 315-330.

[43] K. Szymiczek, Witt equivalence of global fields. Comm. Algebra 19 (1991) 1125-1149.

[44] K. Szymiczek, Witt equivalence of global fields. II. Relative quadratic extensions. Trans. Amer. Math. Soc. 343 (1994) 277-303.

[45] K. Szymiczek, 2-ranks of class groups of Witt equivalent number fields. Number theory (Cieszyn, 1998). Ann. Math. Sil. No. 12 (1998), 53-64.

[46] R. Ware, Valuation rings and rigid elements in fields. Canad. J. Math. 33 (1981), no. 6, $1338-1355$.

[47] L.C. Washington, Class numbers of the simplest cubic fields. Math. Comp. 48 (1987), no. $177,371-384$

[48] E. Witt, Theorie der quadratischen Formen in beliebigen Körpern. Journal für die reine und angewandte Mathematik 176 (1937) 31-44. 
Institute of Mathematics, University of Silesia,

ul. Bankowa 14, 40-007 Katowice, Poland

AND

Department of Computer Science, AGH University of Science and Technology,

AL. Mickiewicza 30, 30-059 Kraków, Poland

E-mail address: pawel.gladki@us.edu.pl

Department of Mathematics and Statistics, University of Saskatchewan,

106 Wiggins Rd., Saskatoon, SK S7N 5E6, Canada

E-mail address: marshall@math.usask.ca 